\title{
Scalar Field Analysis over Point Cloud Data
}

\author{
Frédéric Chazal · Leonidas J. Guibas • \\ Steve Y. Oudot • Primoz Skraba
}

Received: 19 July 2010 / Revised: 13 April 2011 / Accepted: 18 April 2011 /

Published online: 17 May 2011

(C) Springer Science+Business Media, LLC 2011

\begin{abstract}
Given a real-valued function $f$ defined over some metric space $\mathbb{X}$, is it possible to recover some structural information about $f$ from the sole information of its values at a finite set $L \subseteq \mathbb{X}$ of sample points, whose locations are only known through their pairwise distances in $\mathbb{X}$ ? We provide a positive answer to this question. More precisely, taking advantage of recent advances on the front of stability for persistence diagrams, we introduce a novel algebraic construction, based on a pair of nested families of simplicial complexes built on top of the point cloud $L$, from which the persistence diagram of $f$ can be faithfully approximated. We derive from this construction a series of algorithms for the analysis of scalar fields from point cloud data. These algorithms are simple and easy to implement, they have reasonable complexities, and they come with theoretical guarantees. To illustrate the genericity and practicality of the approach, we also present some experimental results obtained in various applications, ranging from clustering to sensor networks.
\end{abstract}

Keywords Persistent homology · Persistence modules · Sampling theory · Vietoris-Rips complexes · Morse theory

F. Chazal · S.Y. Oudot · P. Skraba ( $\varangle)$

Geometrica, INRIA-Saclay, 4 Rue Jacques Monod, 91400 Orsay, France

e-mail: primoz.skraba@inria.fr

F. Chazal

e-mail: frederic.chazal@inria.fr

S.Y. Oudot

e-mail: steve.oudot@inria.fr

L.J. Guibas

Department of Computer Science, Stanford University, Stanford 93405, USA

e-mail: guibas@cs.stanford.edu 


\section{Introduction}

Suppose that we are given a collection of sensors spread out in some planar region, and suppose that these sensors measure some intensive physical quantity, such as temperature or humidity. Assuming that the nodes do not know their geographic location but that they can detect which other nodes lie in their vicinity, is it possible to recover some high-level information about the measured quantity, such as the number of its peaks or valleys, as well as a sense of their prominence? Consider now the case were we are given a finite set of sample points in Euclidean space, drawn from some unknown probability density $f$. Suppose that we can compute at each of these points a rough estimate of the local density. Can we then infer the number of prominent peaks of $f$, which we could later use as the input parameter to a clustering algorithm? Can we tell how to merge the basins of attraction of the maxima, in order to guide the clustering? Consider finally the case where a movie database is provided together with a similarity measure between movies and a measure of popularity for each movie. Can we extract the prominent peaks of the popularity measure, so as to provide information on the general trends of the public's tastes?

These three scenarios are just special instances of a same generic problem: given an unknown domain $\mathbb{X}$ and a scalar field $f: \mathbb{X} \rightarrow \mathbb{R}$ whose values are known only at a finite set $L$ of sample points, the goal is to extract some structural information about $f$ from the sole information of the pairwise distances between the data points and of their function values. In the above scenarios one is mainly interested in finding the peaks and valleys of the function, together with their respective basins of attraction. ${ }^{1}$ In addition, it is desirable to have a mechanism for distinguishing between significant and insignificant peaks or valleys of $f$, which requires to introduce some notion of prominence for the critical points of a function. This is where topological persistence comes into play: inspired from Morse theory, this framework describes the evolution of the topology of the sublevel-sets of $f$, i.e., the sets of type $f^{-1}((-\infty, a])$, as parameter $a$ ranges from $-\infty$ to $+\infty$. Topological changes occur only at critical points of $f$ that are paired in a natural way. For instance, a new connected component appears in $f^{-1}((-\infty, a])$ when $a$ reaches the $f$-value of a local minimum, and this component gets connected to the rest of the sublevel-set as $a$ reaches the $f$-value of a saddle. The outcome of this process is a set of intervals, called a persistence barcode [16], each of which corresponds to a pair of critical points and gives the birth and death times of a homological feature of the sublevel-sets of $f$-see Fig. 1. An equivalent representation is by a multiset of points in the plane, called a persistence diagram, where the coordinates of each point correspond to the endpoints of some interval in the barcode. Such barcodes or diagrams can be used to guide the simplification of the graphs of real-valued functions by iterative cancellations of critical pairs $[20,21]$. As such, they provide the desired information for evaluating the prominence of the peaks and valleys (and in fact of all the critical points) of a scalar field.

Thus, our problem becomes the following: given $\mathbb{X}, f, L$ as above, is it possible to approximate the persistence diagram of $f$ from the pairwise distances between

\footnotetext{
${ }^{1}$ In the context of clustering, this approach to the problem is reminiscent of mode-seeking techniques such as Mean-Shift [17].
} 


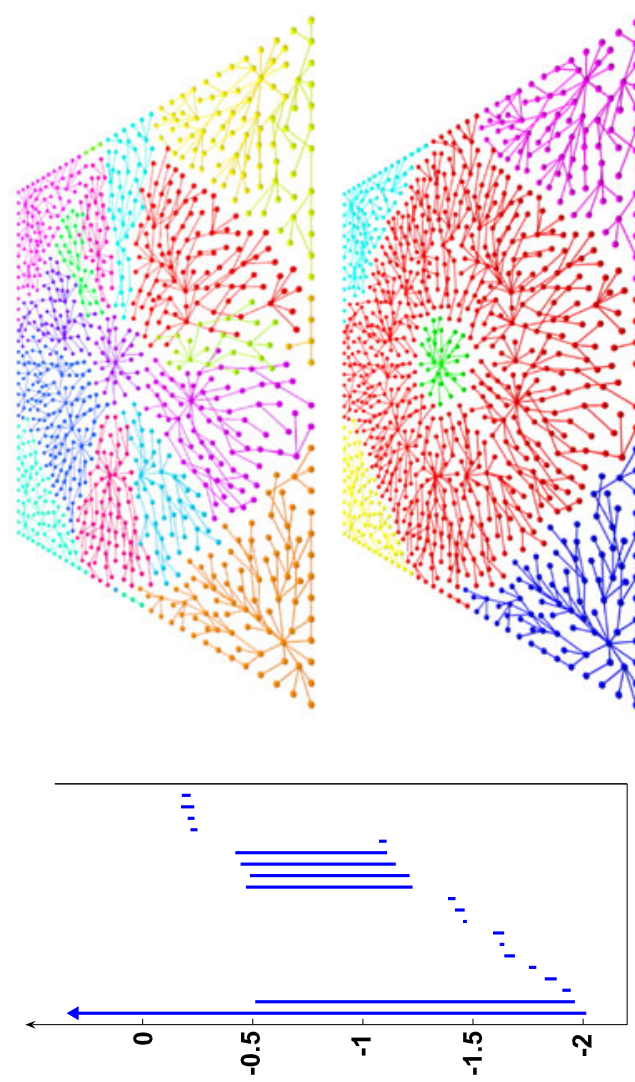

竞

$\stackrel{\because}{\because}$.

茫

$\pi$

苛

要

I

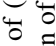

$\approx$ 응

苛

8

ఖ

娄

这

สี

흥

言

武范

$\stackrel{2}{ \pm}$

。

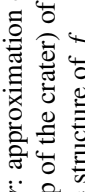

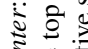

Uั

呟.
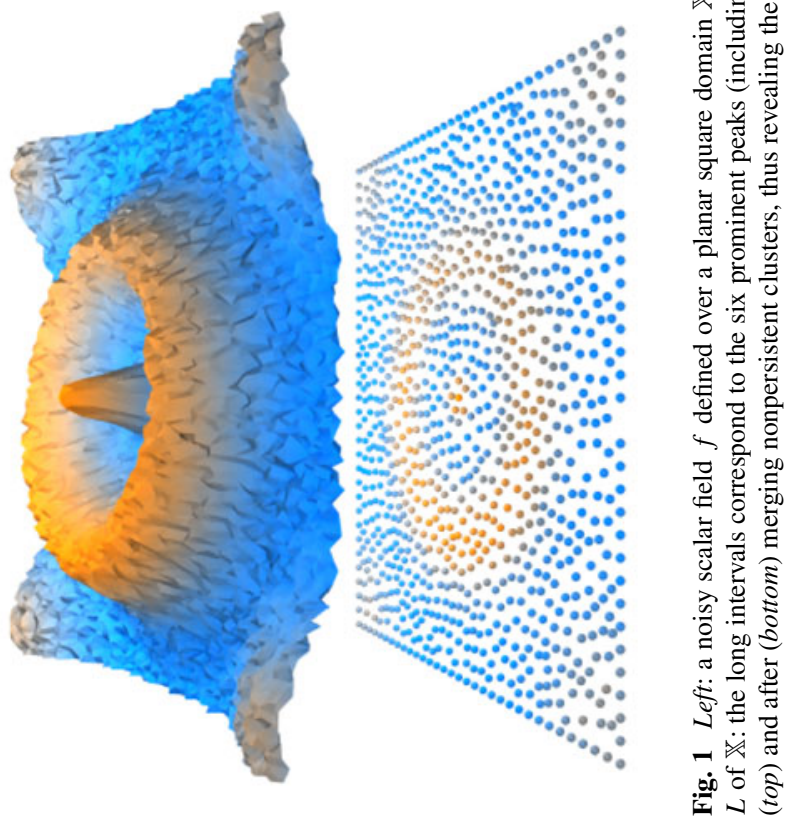
the points of $L$ and from the values of $f$ at these points? The main contribution of the paper is a positive answer to this question. More precisely, in Sect. 3 we exhibit a novel algebraic construction, based on a pair of nested families of simplicial complexes-derived from the so-called (Vietoris-)Rips complexes of L, defined below-from which the persistence diagram of $f$ can be approximated (Theorem 2). We also show the robustness of our construction with respect to noise in the pairwise distances or function values (Theorems 3 and 4). From these structural results we derive algorithms (Sect. 4) for approximating the persistence diagram of $f$ from its values at a finite set of samples, both in static (fixed $f$ ) and in dynamic (timevarying $f$ ) settings. We also give a procedure for finding the basins of attraction of the peaks of $f$ inside the point cloud $L$ and for merging these basins according to the persistence information, as illustrated in Fig. 1 (right). Our algorithms are based on variants $[14,15]$ of the celebrated persistence algorithm. They can be easily implemented, they have reasonable complexities, and they are provably correct for the most part. To illustrate the versatility of the approach, we provide experimental results obtained in a variety of applications (Sect. 5): these illustrate the potential of our method and its general interest. Some of these applications [10, 30] have been worked on since the short-version of this paper [9].

Related Work Topological persistence and its applications have been an extensively studied topic since the introduction of the persistence algorithm by Edelsbrunner et al. [20]. First designed for simplicial complexes in $\mathbb{R}^{3}$, this algorithm was later extended to compute the persistent homology of discrete functions over arbitrary finite simplicial complexes [35]. A number of variants were also proposed, for instance, to cope with changes in the function over time [15] or to handle pairs of functions defined over nested pairs of spaces [14]. All these methods deal with functions defined over simplicial complexes, and in some sense our work suggests a way of extending the approach to a more general class of spaces via finite sampling and modulo some controlled errors in the output.

Topological persistence has already been used in the past for the analysis and simplification of scalar fields. The original persistence paper [20] showed how to simplify the graph of a piecewise-linear (PL) real-valued function $f$ defined over a simplicial complex $\mathbb{X}$ in $\mathbb{R}^{3}$, by iteratively cancelling the pairs of critical points provided by the persistence barcode of $f$. This approach was later refined in the special case where $\mathbb{X}$ is a triangulated 2-manifold to only cancel the pairs corresponding to short intervals in the barcode, thus removing all topological noise up to a certain prescribed amplitude $[2,21]$. In parallel, people have considered computing accurate or simplified representations of Morse-Smale complexes that capture important information about the structure of scalar fields. Indeed, the Morse-Smale complex of a function $f: \mathbb{X} \rightarrow \mathbb{R}$ is a partition of the space $\mathbb{X}$ into regions where the flow induced by the gradient vector field of $f$ is uniform. Building upon the idea of iterative cancellations of pairs of critical points, it is possible to construct hierarchies of increasingly coarse MorseSmale complexes from PL functions defined over triangulated 2- or 3-manifolds [3, $5,19,25,26]$. Although our question of finding the basins of attraction of the peaks of a scalar field may seem a simplistic variant of the above problems, we claim that it is in fact not, as our context is much more general and our knowledge of the function 
$f$ is much weaker. In particular, the resort to a PL approximation of $f$ in our potentially high-dimensional or even non-Euclidean setting would be prohibitively costly, if not impossible. Note also that, in applications such as the scenarios described at the beginning of the section, the knowledge of the basins of attraction of the (significant) peaks of the scalar field is sufficient for further processing.

A last trend of work in which persistence has played a prominent role is homology inference from point cloud data, where the goal is to recover the homological type of an unknown space $\mathbb{X}$ from a finite set $L$ of sample points. The idea is to consider the function distance to $L$, either inside $\mathbb{X}$ or in some ambient space $\mathbb{Y}$ where $\mathbb{X}$ is embedded. Under sufficient sampling density, this function approximates the distance to $\mathbb{X}$, and therefore their persistence diagrams are close, by a stability result due to Cohen-Steiner et al. [13]. Thus, the sole knowledge of the sample points is enough to approximate the persistence diagram of the distance to $\mathbb{X}$, from which the homology of $\mathbb{X}$ is easily inferred $[11,13]$. In practice, however, the cost of estimating the distance to $L$ at every point of $\mathbb{X}$ or of some ambient space $\mathbb{Y}$ is prohibitive, thus requiring the resort to auxiliary algebraic constructions. Among the most popular ones is the Rips complex $R_{\alpha}(L)$, which is the abstract simplicial complex whose simplices correspond to nonempty subsets of $L$ of diameter less than $\alpha$. The building of this complex only involves comparisons of distances, which makes it a good candidate data structure in practice. Furthermore, as proved in [12], a pair of nested Rips complexes $R_{\alpha}(L) \subseteq R_{\beta}(L)$ can provably well capture the homology of the underlying space $\mathbb{X}$, even though none of the individual complexes does. Our algebraic construction (see Sect. 3) is directly inspired from this property, and in fact our theoretical analysis is articulated in the same way as in [12], namely: we first work out structural properties of unions of geodesic balls, which we prove to also hold for their nerves (also called Čech complexes); then, using strong relationships between families of Čech and Rips complexes, we derive structural properties for the latter. Note however that the core of the analysis differs significantly from [12], because our families of complexes are built differently. In particular, the classical notion of stability for persistence diagrams, as introduced in [13], is not broad enough for our setting, where it is replaced by a generalized notion recently proposed by Chazal et al. [6].

\section{Background}

Our analysis uses singular homology with coefficients in a commutative ring $R$, assumed to be a field throughout the paper and omitted in the notation. We also use some elements of Riemannian geometry and Morse theory (mainly in Sect. 4.3). We refer the reader to $[4,27,28]$ for comprehensive introductions to these topics.

\subsection{Geodesic $\varepsilon$-Samples on Riemannian Manifolds}

Throughout the paper, unless otherwise stated, $\mathbb{X}$ denotes a compact Riemannian manifold, possibly with boundary, and $\mathrm{d}_{\mathbb{X}}$ denotes its geodesic distance. Our analysis turns out to hold for a larger class of length spaces; however, for simplicity, we restrict the focus of the paper to the Riemannian setting. Given a point $x \in \mathbb{X}$ and a real value 
$r \geq 0$, let $B_{\mathbb{X}}(x, r)$ denote the open geodesic ball of center $x$ and radius $r$, namely $B_{\mathbb{X}}(x, r)=\left\{y \in \mathbb{X}, \mathrm{d}_{\mathbb{X}}(x, y)<r\right\}$. For all sufficiently small values $r \geq 0$, the ball $B_{\mathbb{X}}(x, r)$ is known to be strongly convex, that is, for every pair of points $y, y^{\prime}$ in the closure of $B_{\mathbb{X}}(x, r)$, there exists a unique shortest path in $\mathbb{X}$ between $y$ and $y^{\prime}$, and the interior of this path is included in $B_{\mathbb{X}}(x, r)$. Let $\varrho(x)>0$ be the supremum of the radii such that this property holds. Since $\mathbb{X}$ is compact, the infimum of $\varrho(x)$ over the points of $\mathbb{X}$ is positive and known as the strong convexity radius of $\mathbb{X}$, denoted $\varrho(\mathbb{X})$. This quantity plays an important role in the paper because strongly convex sets are contractible, ${ }^{2}$ and because intersections of strongly convex sets are also strongly convex.

In the sequel, $L$ denotes a finite set of points of $\mathbb{X}$ that form a geodesic $\varepsilon$-sample of $\mathbb{X}$, namely, for all $x \in \mathbb{X}, \mathrm{d}_{\mathbb{X}}(x, L)<\varepsilon$. Here, parameter $\varepsilon$ is homogeneous to a length, and it controls the density of the point cloud $L$. Our theoretical claims will assume this density to be high enough, via a condition on $\varepsilon$ stipulating that the latter is at most a fraction of the strong convexity radius of $\mathbb{X}$.

\subsection{Persistence Modules and Filtrations}

The main algebraic objects under study here are persistence modules. A persistence module is a family $\left\{\Phi_{\alpha}\right\}_{\alpha \in \mathbb{R}}$ of $R$-modules together with a family $\left\{\phi_{\alpha}^{\beta}: \Phi_{\alpha} \rightarrow\right.$ $\left.\Phi_{\beta}\right\}_{\alpha \leq \beta \in \mathbb{R}}$ of homomorphisms such that for all $\alpha \leq \beta \leq \gamma, \phi_{\alpha}^{\gamma}=\phi_{\beta}^{\gamma} \circ \phi_{\alpha}^{\beta}$ and $\phi_{\alpha}^{\alpha}=\operatorname{id}_{\Phi_{\alpha}}$. Persistence modules are often derived from filtrations that are families $\left\{F_{\alpha}\right\}_{\alpha \in \mathbb{R}}$ of topological spaces that are nested with respect to inclusion. For all $\alpha \leq \beta$, the canonical inclusion map $F_{\alpha} \hookrightarrow F_{\beta}$ induces homomorphisms between the homology groups $H_{k}\left(F_{\alpha}\right) \rightarrow H_{k}\left(F_{\beta}\right)$ of all dimensions $k \in \mathbb{N}$. Thus, for any fixed $k$, the family $\left\{H_{k}\left(F_{\alpha}\right)\right\}_{\alpha \in \mathbb{R}}$ forms a persistence module, called $k$ th persistent homology module of $\left\{F_{\alpha}\right\}_{\alpha \in \mathbb{R}}$, where the homomorphisms between $R$-modules are understood to be those induced by inclusions.

An important class of filtrations are the ones formed by the sublevel-sets of real-valued functions. Given a topological space $\mathbb{X}$ and a function $f: \mathbb{X} \rightarrow \mathbb{R}$, the sublevel-sets filtration of $f$ is the family $\left\{F_{\alpha}\right\}_{\alpha \in \mathbb{R}}$ of subspaces of $\mathbb{X}$ of type $F_{\alpha}=f^{-1}((-\infty, \alpha])$. This family forms a filtration because $f^{-1}((-\infty, \alpha]) \subseteq$ $f^{-1}((-\infty, \beta])$ whenever $\alpha \leq \beta$. A real-valued function that has played a prominent role in homology inference is the geodesic distance to a finite point cloud $L$. The 0 -sublevel set of this function is $L$ itself, while for any $\alpha>0$, its $\alpha$-sublevel set is the closure of the so-called $\alpha$-offset $L^{\alpha}$, defined as the union of the open geodesic balls of radius $\alpha$ about the points of $L$, namely, $L^{\alpha}=\bigcup_{p \in L} B_{\mathbb{X}}(p, \alpha)$. Important structural properties of growing families of open balls, some of which will be exploited in Sect. 3.1 of this paper, follow from the properties of the sublevel-sets of the distance function $[8,29]$.

Since the offsets of a point cloud $L$ can be difficult to manipulate, they are often replaced by purely combinatorial constructions in practice. A natural choice is to use the nerve of the family of open geodesic balls used in the definition of the $\alpha$-offset of $L$. Specifically, the nerve of the family $\left\{B_{\mathbb{X}}(p, \alpha)\right\}_{p \in L}$ is the abstract simplicial

\footnotetext{
${ }^{2} \mathrm{~A}$ topological space is contractible if it can be continuously deformed to a point within itself.
} 
complex of vertex set $L$ whose simplices correspond to nonempty subsets of the family whose elements have a nonempty common intersection. This complex is also known as the Čech complex and denoted $C_{\alpha}(L)$. Thanks to the duality that exists between unions of open balls and their nerves (see Lemma 3 below), Čech complexes $C_{\alpha}(L)$ enjoy many interesting properties that will be exploited as well in Sect. 3.1.

An even simpler combinatorial construction is the so-called (Vietoris-)Rips complex $R_{\alpha}(L)$, which is the abstract simplicial complex of vertex set $L$ whose simplices correspond to nonempty subsets of $L$ of geodesic diameter less than $\alpha$. The building of this complex only involves comparisons of distances, which makes it a good candidate data structure in practice. Furthermore, Rips complexes are known to be closely related to Čech complexes through the following sequence of inclusions, which holds in any arbitrary metric space (see, e.g., [12]):

$$
\forall \alpha>0, \quad C_{\frac{\alpha}{2}}(L) \subseteq R_{\alpha}(L) \subseteq C_{\alpha}(L) .
$$

Several other combinatorial constructions, such as the $\alpha$-shape or the witness complex, have been proven to be useful in the context of homology inference. These will not be considered in the paper.

Finally, let us mention that the above constructions are parameterized by a unique quantity $\alpha$, which one usually lets vary from 0 to $+\infty$ to get a filtration. In contrast, our filtrations will be obtained by fixing $\alpha$ to some constant value and by letting the vertex set grow from $\emptyset$ to $L$.

\subsection{Persistence Diagrams and Stability}

Persistence diagrams have been introduced as a succinct way of describing the algebraic structure of a persistence module [35]. There is a restriction though: without any further assumptions, the algebraic structure of a persistence module can be arbitrarily complicated, thereby making it impossible to find a descriptor that is both succinct and complete. This is where the concept of tameness ${ }^{3}$ comes into play:

Definition 1 A persistence module $\left(\left\{\Phi_{\alpha}\right\}_{\alpha \in \mathbb{R}},\left\{\phi_{\alpha}^{\beta}\right\}_{\alpha \leq \beta \in \mathbb{R}}\right)$ is tame if for all $\alpha<\beta$, $\operatorname{rank} \phi_{\alpha}^{\beta}<+\infty$.

This condition is restrictive enough for persistence diagrams to be well defined; yet the concept of tameness remains sufficiently wide to encompass a large class of persistence modules. In particular, all persistent homology modules of nested families of finite simplicial complexes are tame. As a consequence, all persistence modules introduced in Sects. 3 and 4 of this paper will be tame.

Following [6], the persistence diagram of a tame persistence module $\left(\left\{\Phi_{\alpha}\right\}_{\alpha \in \mathbb{R}}\right.$, $\left.\left\{\phi_{\alpha}^{\beta}\right\}_{\alpha \leq \beta \in \mathbb{R}}\right)$ is defined as a multiset of points in the extended plane $\overline{\mathbb{R}}^{2}$, where $\overline{\mathbb{R}}=\mathbb{R} \cup\{-\infty,+\infty\}$. This multiset is obtained as the limit of the following iterative process: given arbitrary values $a, \varepsilon>0$, we discretize the persistence module over the integer scale $a+\varepsilon \mathbb{Z}$, considering the subfamily $\left\{\Phi_{a+k \varepsilon}\right\}_{k \in \mathbb{Z}}$ of vector spaces

\footnotetext{
${ }^{3}$ We borrow this concept from [6], where it is called 0-tameness and made weaker than in [13].
} 
together with the subfamily $\left\{\phi_{a+k \varepsilon}^{a+l \varepsilon}\right\}_{k \leq l \in \mathbb{Z}}$ of linear maps. Its persistence diagram is defined naturally ${ }^{4}$ as the set of vertices of the regular grid $(a+\varepsilon \mathbb{Z}) \times(a+\varepsilon \mathbb{Z})$ in $\overline{\mathbb{R}}^{2}$, plus the diagonal $\Delta=\{(x, x), x \in \overline{\mathbb{R}}\}$, where each grid vertex $(a+k \varepsilon, a+l \varepsilon)$ is given the (finite) multiplicity

$\operatorname{mult}(a+k \varepsilon, a+l \varepsilon)=\operatorname{rank} \phi_{a+k \varepsilon}^{a+(l-1) \varepsilon}-\operatorname{rank} \phi_{a+k \varepsilon}^{a+l \varepsilon}+\operatorname{rank} \phi_{a+(k-1) \varepsilon}^{a+l \varepsilon}-\operatorname{rank} \phi_{a+(k-1) \varepsilon}^{a+(l-1) \varepsilon}$, while each point of $\Delta$ is given infinite multiplicity. Then, the persistence diagram of $\left(\left\{\Phi_{\alpha}\right\}_{\alpha \in \mathbb{R}},\left\{\phi_{\alpha}^{\beta}\right\}_{\alpha \leq \beta \in \mathbb{R}}\right)$ is the limit multiset obtained as $\varepsilon \rightarrow 0$, which is known to be independent of the choice of $a[6]$.

An important property of persistence diagrams is their stability under small perturbations. Cohen-Steiner et al. [13] proposed the first result in this vein: given two tame continuous real-valued functions $f, g$ defined over a same triangulable space $\mathbb{X}$, for all $k \in \mathbb{N}$, the bottleneck distance between the persistence diagrams of the $k$ th persistent homology modules of their sublevel-sets filtrations is at most $\sup _{x \in \mathbb{X}} \mid f(x)-$ $g(x) \mid$. Recall that the bottleneck distance $\mathrm{d}_{B}^{\infty}(A, B)$ between two multisets in $\overline{\mathbb{R}}^{2}$ endowed with the $l^{\infty}$-norm is the quantity $\min _{\gamma} \max _{p \in A}\|p-\gamma(p)\|_{\infty}$, where $\gamma$ ranges over all bijections from $A$ to $B$.

Recently, Chazal et al. [6] extended the result of [13] by dropping the continuity and triangulability assumptions, as well as the functional setting. To do so, they had to introduce a new notion of proximity between persistence modules:

Definition 2 Two persistence modules $\left(\left\{\Phi_{\alpha}\right\}_{\alpha \in \mathbb{R}},\left\{\phi_{\alpha}^{\beta}\right\}_{\alpha \leq \beta \in \mathbb{R}}\right)$ and $\left(\left\{\Psi_{\alpha}\right\}_{\alpha \in \mathbb{R}}\right.$, $\left\{\psi_{\alpha}^{\beta}\right\}_{\alpha \leq \beta \in \mathbb{R}}$ ) are (strongly) $\varepsilon$-interleaved if there exist two families of homomorphisms, $\left\{\mu_{\alpha}: \Phi_{\alpha} \rightarrow \Psi_{\alpha+\varepsilon}\right\}_{\alpha \in \mathbb{R}}$ and $\left\{v_{\alpha}: \Psi_{\alpha} \rightarrow \Phi_{\alpha+\varepsilon}\right\}_{\alpha \in \mathbb{R}}$, that make the following diagrams commute for all values $\alpha \leq \beta \in \mathbb{R}$ :
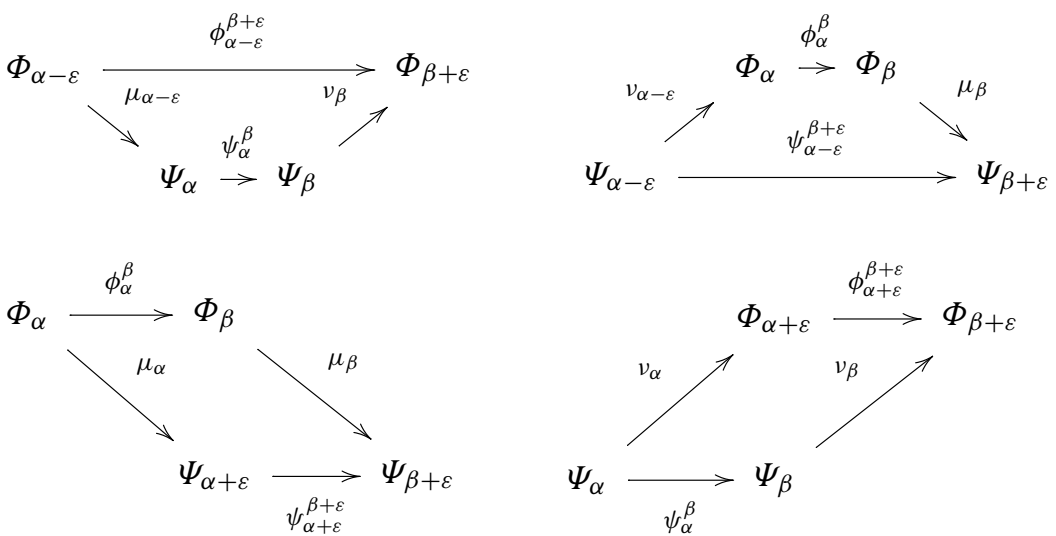

Under these conditions, Chazal et al. proved the following generalized stability result [6]:

\footnotetext{
${ }^{4}$ In the particular case of a discretized persistence module, this definition does coincide with the one of [13]. 
Theorem 1 If two tame persistence modules are $\varepsilon$-interleaved, then, in the extended plane $\overline{\mathbb{R}}^{2}$ endowed with the $l^{\infty}$-norm, the bottleneck distance between their persistence diagrams is at most $\varepsilon$.

An important special case is that of the $k$ th persistent homology modules of two filtrations $\left\{F_{\alpha}\right\}_{\alpha \in \mathbb{R}}$ and $\left\{G_{\alpha}\right\}_{\alpha \in \mathbb{R}}$ that are $\varepsilon$-interleaved w.r.t. inclusion, that is, $F_{\alpha} \subseteq G_{\alpha+\varepsilon}$ and $G_{\alpha} \subseteq F_{\alpha+\varepsilon}$ for all $\alpha \in \mathbb{R}$. In this case, the maps $\mu_{\alpha}$ and $\nu_{\alpha}$ induced at homology level by the canonical inclusions $F_{\alpha} \hookrightarrow G_{\alpha+\varepsilon}$ and $G_{\alpha} \hookrightarrow F_{\alpha+\varepsilon}$ make the diagrams of (2) and (3) commute, thus $\varepsilon$-interleaving the $k$ th persistent homology modules of the two filtrations. Theorem 1 guarantees then that their persistence diagrams are $\varepsilon$-close in the bottleneck distance.

Note About the Exposition In order to simplify the exposition in the sequel, we allow ourselves some degree of sloppiness in the notation. Specifically, we omit the ranges of the indices when these are obvious, thus designating a filtration of parameter $\alpha \in \mathbb{R}$ by $\left\{F_{\alpha}\right\}$ and a persistence module of parameters $\alpha \leq \beta \in \mathbb{R}$ by $\left(\left\{\Phi_{\alpha}\right\},\left\{\phi_{\alpha}^{\beta}\right\}\right)$. In addition, we use the following shortcuts: the persistence diagram of the $k$ th persistent homology module of a filtration $\left\{F_{\alpha}\right\}$ is simply called the $k$ th persistence diagram of $\left\{F_{\alpha}\right\}$. Furthermore, the filtration itself is said to be tame if its $k$ th persistent homology module is tame for all values $k \in \mathbb{N}$. At a higher level, the $k$ th persistent homology module of a real-valued function $f$ refers by default to the $k$ th persistent homology module of its sublevel-sets filtration, and $f$ is said to be tame if its sublevel-sets filtration is tame. Finally, the $k$ th persistence diagram of $f$ is the $k$ th persistence diagram of its sublevel-sets filtration.

\section{Structural Properties}

Let $\mathbb{X}$ be a compact Riemannian manifold, possibly with boundary, and let $f: \mathbb{X} \rightarrow$ $\mathbb{R}$ be a $c$-Lipschitz function. As we will see in Lemma 4 below, these assumptions automatically imply that $f$ is tame. Assuming $\mathbb{X}$ and $f$ to be unknown, we want to approximate the $k$ th persistence diagram of $f$ from the values of the function at a finite set $L$ of sample points that form a geodesic $\varepsilon$-sample of $\mathbb{X}$. Our main result (Theorem 2 below) claims that this is possible using an algebraic construction based on Rips complexes. The main advantage of this construction is that it leads to an easyto-compute data structure, which will be described in the algorithms section (Sect. 4). From now on, $L_{\alpha}$ denotes the set $L \cap f^{-1}((-\infty, \alpha])$.

Our construction is inspired from [12], where it is shown that a pair of nested Rips complexes can provably well capture the homology of a domain even though none of the individual Rips complexes does. Given a fixed parameter $\delta>0$, we use two Ripsbased filtrations simultaneously, $\left\{R_{\delta}\left(L_{\alpha}\right)\right\}_{\alpha \in \mathbb{R}}$ and $\left\{R_{2 \delta}\left(L_{\alpha}\right)\right\}_{\alpha \in \mathbb{R}}$, and we consider the persistence modules formed at homology level by the images of the homomorphisms induced by the inclusions $R_{\delta}\left(L_{\alpha}\right) \hookrightarrow R_{2 \delta}\left(L_{\alpha}\right)$. Specifically, for all $k \in \mathbb{N}$ and 
all $\alpha \leq \beta$, we have the following induced commutative diagram at homology level:

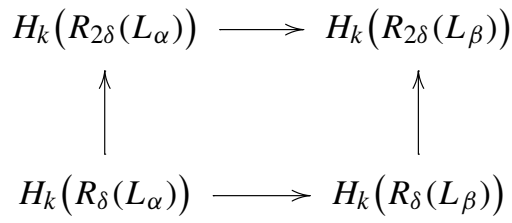

Letting $\Phi_{\alpha}$ be the image of $H_{k}\left(R_{\delta}\left(L_{\alpha}\right)\right) \rightarrow H_{k}\left(R_{2 \delta}\left(L_{\alpha}\right)\right)$, we get that the above commutative diagram induces a map $\phi_{\alpha}^{\beta}: \Phi_{\alpha} \rightarrow \Phi_{\beta}$. Since this is true for all $\alpha \leq \beta$, the family $\left\{\Phi_{\alpha}\right\}_{\alpha \in \mathbb{R}}$ of vector spaces, together with the family $\left\{\phi_{\alpha}^{\beta}\right\}_{\alpha \leq \beta}$ of linear maps, forms a persistence module. By analogy with the terminology of Sect. 2, we call it the $k$ th persistent homology module of the nested pair of filtrations $\left\{R_{\delta}\left(L_{\alpha}\right) \hookrightarrow R_{2 \delta}\left(L_{\alpha}\right)\right\}_{\alpha \in \mathbb{R}}$, and its persistence diagram the $k$ th persistence diagram of the nested pair. This construction is in fact not specific to families of Rips complexes, and it allows us to define a persistence module $\left(\left\{\Phi_{\alpha}\right\},\left\{\phi_{\alpha}^{\beta}\right\}\right)$ from the $k$-dimensional homology groups of any pair of filtrations $\left\{G_{\alpha}\right\}$ and $\left\{G_{\alpha}^{\prime}\right\}$ that is nested with respect to inclusion: $\forall \alpha \in \mathbb{R}, G_{\alpha} \subseteq G_{\alpha}^{\prime}$.

Theorem 2 Let $\mathbb{X}$ be a compact Riemannian manifold, possibly with boundary, and let $f: \mathbb{X} \rightarrow \mathbb{R}$ be a c-Lipschitz function. Let also L be a geodesic $\varepsilon$-sample of $\mathbb{X}$. If $\varepsilon<\frac{1}{4} \varrho(\mathbb{X})$, then for any $\delta \in\left[2 \varepsilon, \frac{1}{2} \varrho(\mathbb{X})\right)$ and for any $k \in \mathbb{N}$, the kth persistent homology modules of $f$ and of the nested pair of filtrations $\left\{R_{\delta}\left(L_{\alpha}\right) \hookrightarrow R_{2 \delta}\left(L_{\alpha}\right)\right\}_{\alpha \in \mathbb{R}}$ are $2 c \delta$-interleaved. Therefore, the bottleneck distance between their persistence diagrams is at most $2 c \delta$, by Theorem 1.

In practice, the $k$ th persistent homology module of the pair of filtrations $\left\{R_{\delta}\left(L_{\alpha}\right)\right.$ $\left.\hookrightarrow R_{2 \delta}\left(L_{\alpha}\right)\right\}_{\alpha \in \mathbb{R}}$ does not have to be built explicitly since its persistence diagram can be computed directly from the filtrations $\left\{R_{\delta}\left(L_{\alpha}\right)\right\}_{\alpha \in \mathbb{R}}$ and $\left\{R_{2 \delta}\left(L_{\alpha}\right)\right\}_{\alpha \in \mathbb{R}}$ [14]. The next two sections are devoted to the proof of Theorem 2 . The core argument, based on a technique of algebraic topology called diagram chasing, is presented in Sect. 3.2. It makes use of preliminary results on unions of balls and their nerves, introduced in Sect. 3.1. Finally, Sect. 3.3 addresses the robustness of our main result with respect to small perturbations of the geodesic distances or function values.

\subsection{Preliminaries: Unions of Geodesic Balls and Their Nerves}

Let $\delta>0$ be a fixed parameter. Consider the filtration $\left\{L_{\alpha}^{\delta}\right\}_{\alpha \in \mathbb{R}}$ formed by the $\delta$-offsets of the subsets $L_{\alpha}$. Recall that the $\delta$-offset of $L_{\alpha}$ is defined by $L_{\alpha}^{\delta}=$ $\bigcup_{p \in L_{\alpha}} B_{\mathbb{X}}(p, \delta)$.

Lemma 1 Let $\mathbb{X}, f, L$ be as in Theorem 2. Then, for any $\delta \geq \varepsilon$, the sublevel-sets filtration $\left\{F_{\alpha}\right\}$ of $f$ and the filtration $\left\{L_{\alpha}^{\delta}\right\}_{\alpha \in \mathbb{R}}$ are $c \delta$-interleaved w.r.t. inclusion. Hence, for all $k \in \mathbb{N}$, the bottleneck distance between their $k$ th persistence diagrams is at most $c \delta$, by Theorem 1 . 
Proof Consider an arbitrary value $\alpha \in \mathbb{R}$ and take a point $p \in F_{\alpha}$. Since $L$ is a geodesic $\varepsilon$-sample of $\mathbb{X}$, there exists some point $q \in L$ such that $\mathrm{d}_{\mathbb{X}}(p, q)<\varepsilon \leq \delta$. Since $f$ is $c$-Lipschitz, we have $f(q) \leq f(p)+c \delta \leq \alpha+c \delta$, which implies that $q \in L \cap F_{\alpha+c \delta}$. Hence, $p$ belongs to $L_{\alpha+c \delta}^{\delta}$. Reciprocally, take a point $p \in L_{\alpha}^{\delta}$. By definition, there exists some point $q \in L_{\alpha}$ such that $\mathrm{d}_{\mathbb{X}}(p, q)<\delta$. Since $f$ is $c$-Lipschitz, we have $f(p) \leq f(q)+c \delta \leq \alpha+c \delta$. Therefore, $p$ belongs to $F_{\alpha+c \delta}$. This proves that $\left\{F_{\alpha}\right\}$ and $\left\{L_{\alpha}^{\delta}\right\}$ are $c \delta$-interleaved w.r.t. inclusion.

We now turn our focus to the nerves $C_{\delta}\left(L_{\alpha}\right)$ of the offsets $L_{\alpha}^{\delta}$ :

Lemma 2 Let $\mathbb{X}, f, L$ be as in Theorem 2. For all $k \in \mathbb{N}$, there exists a family of isomorphisms $\left\{H_{k}\left(C_{\delta}\left(L_{\alpha}\right)\right) \rightarrow H_{k}\left(L_{\alpha}^{\delta}\right)\right\}_{\alpha \in \mathbb{R}, \delta<\varrho(\mathbb{X})}$ such that the following diagrams (where horizontal homomorphisms are induced by inclusions) commute: $\forall \alpha \leq \beta \in \mathbb{R}$, $\forall \delta \leq \xi<\varrho(\mathbb{X})$

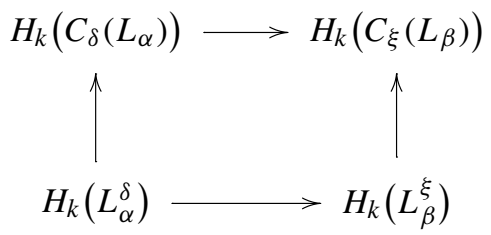

As a consequence, $\forall k \in \mathbb{N}, \forall \delta<\varrho(\mathbb{X})$, the $k$ th persistence diagrams of $\left\{C_{\delta}\left(L_{\alpha}\right)\right\}_{\alpha \in \mathbb{R}}$ and $\left\{L_{\alpha}^{\delta}\right\}_{\alpha \in \mathbb{R}}$ are identical.

The proof of this result, detailed below, relies on the following technical lemma ${ }^{5}$ from [12], which relates good open covers to their nerves. Given a topological space $\mathbb{X}$ and a family $\left\{U_{a}\right\}_{a \in A}$ of open subsets covering $\mathbb{X}$, the family defines a good cover if, for every finite subset $S$ of $A$, the common intersection $\bigcap_{a \in S} U_{a}$ is either empty or contractible.

Lemma 3 (Lemma 3.4 of [12]) Let $\mathbb{X} \subseteq \mathbb{X}^{\prime}$ be two paracompact spaces, and let $\mathcal{U}=$ $\left\{U_{a}\right\}_{a \in A}$ and $\mathcal{U}^{\prime}=\left\{U_{a^{\prime}}^{\prime}\right\}_{a^{\prime} \in A^{\prime}}$ be good open covers of $\mathbb{X}$ and $\mathbb{X}^{\prime}$, respectively, based on finite parameter sets $A \subseteq A^{\prime}$, such that $U_{a} \subseteq U_{a}^{\prime}$ for all $a \in A$. Then, the homotopy equivalences $\mathcal{N U} \rightarrow \mathbb{X}$ and $\mathcal{N U}^{\prime} \rightarrow \mathbb{X}^{\prime}$ provided by the Nerve Theorem $[27, \S 4 G]$ commute with the canonical inclusions $\mathbb{X} \hookrightarrow \mathbb{X}^{\prime}$ and $\mathcal{N U} \hookrightarrow \mathcal{N U}$ at homology level.

Proof of Lemma 2 Let $k \in \mathbb{N}$. We claim that, for all $\alpha \in \mathbb{R}$ and $\delta<\varrho(\mathbb{X})$, the family of open balls $\left\{B_{\mathbb{X}}(p, \delta)\right\}_{p \in L_{\alpha}}$ forms a good open cover of the set $L_{\alpha}^{\delta}$, that is, for all $l \in \mathbb{N}$ and $p_{1}, \ldots, p_{l} \in L_{\alpha}$, the intersection $I=B_{\mathbb{X}}\left(p_{1}, \delta\right) \cap \cdots \cap B_{\mathbb{X}}\left(p_{l}, \delta\right)$ is either empty or contractible. Indeed, assuming that $I$ is nonempty, we have that each ball

\footnotetext{
${ }^{5}$ Note that the statement of Lemma 3.4 of [12] assumes the parameter sets $A, A^{\prime}$ to be equal. However, the proof of the lemma only uses the facts that $A \subseteq A^{\prime}$ and that the cover $\mathcal{U}$ is subordinate to the cover $\mathcal{U}^{\prime}$ on its own index set $A$. In addition, the statement of the lemma does not specify that the homotopy equivalences considered are the ones provided by the Nerve Theorem $[27, \S 4 \mathrm{G}]$, but this appears clearly in the proof of the lemma.
} 
$B\left(p_{i}, \delta\right)$ is strongly convex because $\delta<\varrho(\mathbb{X})$. As a consequence, $I$ itself is strongly convex and therefore contractible, as mentioned in Sect. 2.1. Thus, $\left\{B_{\mathbb{X}}(p, \delta)\right\}_{p \in L_{\alpha}}$ forms a good open cover of $L_{\alpha}^{\delta}$. Since this is true for all $\alpha \in \mathbb{R}$ and $\delta<\varrho(\mathbb{X})$, Lemma 3 guarantees that the diagram of (4) commutes for any $k \in \mathbb{N}$.

Letting now $\delta=\xi<\varrho(\mathbb{X})$ be fixed, the commutativity of (4) implies that the homomorphisms $H_{k}\left(C_{\delta}\left(L_{\alpha}\right)\right) \rightarrow H_{k}\left(C_{\delta}\left(L_{\beta}\right)\right)$ and $H_{k}\left(L_{\alpha}^{\delta}\right) \rightarrow H_{k}\left(L_{\beta}^{\delta}\right)$ have same rank for all values $\alpha \leq \beta$. Therefore, the filtrations $\left\{C_{\delta}\left(L_{\alpha}\right)\right\}_{\alpha \in \mathbb{R}}$ and $\left\{L_{\alpha}^{\delta}\right\}_{\alpha \in \mathbb{R}}$ have identical $k$ th persistence diagrams.

With these preliminary results at hand, we can now proceed to the proof of Theorem 2. But before, let us mention an interesting side result that explains why the tameness condition on the input function $f$ is not necessary in our context:

Lemma 4 Let $\mathbb{X}$ be a compact Riemannian manifold, possibly with boundary, and let $f: \mathbb{X} \rightarrow \mathbb{R}$ be Lipschitz-continuous. Then, for any $k \in \mathbb{N}$, the $k$ th persistent homology module of $f$ is tame in the sense of Definition 1.

Proof Let $c \geq 0$ denote the Lipschitz constant of $f$. Given any $\alpha<\beta \in \mathbb{R}$, consider a finite point cloud $L \subseteq \mathbb{X}$ that forms a geodesic $r$-sample of $\mathbb{X}$ for some positive $r<\min \left\{\varrho(\mathbb{X}), \frac{\beta-\alpha}{2 c}\right\}$. Such a finite sampling always exists because $r$ is positive and $\mathbb{X}$ is compact. Now, Lemma 1 and our upper bound on $r$ imply that $F_{\alpha} \subseteq L_{\alpha+c r}^{r} \subseteq$ $L_{\beta-c r}^{r} \subseteq F_{\beta}$. Thus, the canonical inclusion $F_{\alpha} \hookrightarrow F_{\beta}$ factors through $L_{\alpha+c r}^{r}$, which implies at $k$ th homology level that $\operatorname{rank} H_{k}\left(F_{\alpha}\right) \rightarrow H_{k}\left(F_{\beta}\right) \leq \operatorname{dim} H_{k}\left(L_{\alpha+c r}^{r}\right)$. Now, since $r<\varrho(\mathbb{X})$, the Nerve theorem [27, §4G] ensures that the union of geodesic balls $L_{\alpha+c r}^{r}$ is homotopy equivalent to its nerve, which is a finite simplicial complex and therefore has finite-dimensional homology groups. As a result, at $k$ th homology level we have rank $H_{k}\left(F_{\alpha}\right) \rightarrow H_{k}\left(F_{\beta}\right) \leq \operatorname{dim} H_{k}\left(L_{\alpha+c r}^{r}\right)<+\infty$.

\subsection{Proof of Theorem 2}

We will in fact prove the following more general (yet technical) result:

Lemma 5 Let $\mathbb{X}, f, L$ be as in Theorem 2. Suppose that there exist $\varepsilon^{\prime} \leq \varepsilon^{\prime \prime} \in$ $[\varepsilon, \varrho(\mathbb{X}))$ and two filtrations, $\left\{G_{\alpha}\right\}$ and $\left\{G_{\alpha}^{\prime}\right\}$, such that for all $\alpha \in \mathbb{R}, C_{\varepsilon}\left(L_{\alpha}\right) \subseteq G_{\alpha} \subseteq$ $C_{\varepsilon^{\prime}}\left(L_{\alpha}\right) \subseteq G_{\alpha}^{\prime} \subseteq C_{\varepsilon^{\prime \prime}}\left(L_{\alpha}\right)$. Then, for all $k \in \mathbb{N}$, the kth persistent homology modules of $f$ and of the nested pair of filtrations $\left\{G_{\alpha} \hookrightarrow G_{\alpha}^{\prime}\right\}_{\alpha \in \mathbb{R}}$ are c $\varepsilon^{\prime \prime}$-interleaved.

Applying Lemma 5 with $\varepsilon^{\prime}=\delta, \varepsilon^{\prime \prime}=2 \delta, G_{\alpha}=R_{\delta}\left(L_{\alpha}\right)$, and $G_{\alpha}^{\prime}=R_{2 \delta}\left(L_{\alpha}\right)$ gives Theorem 2, the sequence of inclusions assumed in the statement of Lemma 5 being ensured by (1) in this case. Lemma 5 itself will be instrumental in Sect. 3.3, in proving the robustness of our main result with respect to small perturbations of geodesic distances.

Proof of Lemma 5 Let $k \in \mathbb{N}$. For clarity, we call $\left(\left\{\Phi_{\alpha}\right\},\left\{\phi_{\alpha}^{\beta}\right\}\right)$ the $k$ th persistent homology module of the nested pair of filtrations $\left\{G_{\alpha} \hookrightarrow G_{\alpha}^{\prime}\right\}_{\alpha \in \mathbb{R}}$, and $\left(\left\{\Psi_{\alpha}\right\},\left\{\psi_{\alpha}^{\beta}\right\}\right)$ the $k$ th persistent homology module of $f$. 
On the one hand, for all values $\alpha \leq \beta$, the sequence of inclusions assumed in the statement of the lemma induces the following commutative diagram at homology level:

$$
\begin{aligned}
& H_{k}\left(C_{\varepsilon}\left(L_{\beta}\right)\right) \stackrel{a_{\beta}}{\longrightarrow} H_{k}\left(G_{\beta}\right) \stackrel{b_{\beta}}{\longrightarrow} H_{k}\left(C_{\varepsilon^{\prime}}\left(L_{\beta}\right)\right) \stackrel{d_{\beta}}{\longrightarrow} H_{k}\left(G_{\beta}^{\prime}\right) \stackrel{e_{\beta}}{\longrightarrow} H_{k}\left(C_{\varepsilon^{\prime \prime}}\left(L_{\beta}\right)\right) \\
& \begin{array}{ccccc}
\uparrow i_{\alpha}^{\beta} & \uparrow_{j \alpha}^{\beta} & \uparrow l_{\alpha}^{\beta} & \uparrow m_{\alpha}^{\beta} & \uparrow n_{\alpha}^{\beta} \\
H_{k}\left(C_{\varepsilon}\left(L_{\alpha}\right)\right) \stackrel{a_{\alpha}}{\longrightarrow} H_{k}\left(G_{\alpha}\right) \stackrel{b_{\alpha}}{\longrightarrow} H_{k}\left(C_{\varepsilon^{\prime}}\left(L_{\alpha}\right)\right) \stackrel{e_{\alpha}}{\longrightarrow} H_{k}\left(G_{\alpha}^{\prime}\right) \stackrel{e_{\alpha}}{\longrightarrow} H_{k}\left(C_{\varepsilon^{\prime \prime}}\left(L_{\alpha}\right)\right)
\end{array}
\end{aligned}
$$

This diagram encodes important relations between the persistence module $\left(\left\{\Phi_{\alpha}\right\}\right.$, $\left.\left\{\phi_{\alpha}^{\beta}\right\}\right)$ and the homology groups of the Čech complexes. It implies, for instance, that the rank of $\phi_{\alpha}^{\beta}$ is at most the rank of $H_{k}\left(C_{\mathcal{E}^{\prime}}\left(L_{\alpha}\right)\right) \rightarrow H_{k}\left(C_{\varepsilon^{\prime}}\left(L_{\beta}\right)\right)$. Indeed, by definition, $\phi_{\alpha}^{\beta}: \Phi_{\alpha} \rightarrow \Phi_{\beta}$ is the restriction of $m_{\alpha}^{\beta}$ to im $d_{\alpha} \circ b_{\alpha}$, and therefore we have $\operatorname{im} \phi_{\alpha}^{\beta}=\operatorname{im} m_{\alpha}^{\beta} \circ d_{\alpha} \circ b_{\alpha}=\operatorname{im} d_{\beta} \circ l_{\alpha}^{\beta} \circ b_{\alpha}$, which implies that $\operatorname{rank} \phi_{\alpha}^{\beta}=$ $\operatorname{rank} d_{\beta} \circ l_{\alpha}^{\beta} \circ b_{\alpha} \leq \operatorname{rank} l_{\alpha}^{\beta}$. Similarly, the rank of $H_{k}\left(C_{\varepsilon}\left(L_{\alpha}\right)\right) \rightarrow H_{k}\left(C_{\varepsilon^{\prime \prime}}\left(L_{\beta}\right)\right)$ is equal to rank $e_{\beta} \circ\left(m_{\alpha}^{\beta} \circ d_{\alpha} \circ b_{\alpha}\right) \circ a_{\alpha} \leq \operatorname{rank} m_{\alpha}^{\beta} \circ d_{\alpha} \circ b_{\alpha}=\operatorname{rank} \phi_{\alpha}^{\beta}$. Thus, for all $\alpha \leq \beta$, the rank of the homomorphism $\phi_{\alpha}^{\beta}$ is sandwiched between the ranks of $H_{k}\left(C_{\varepsilon}\left(L_{\alpha}\right)\right) \rightarrow H_{k}\left(C_{\varepsilon^{\prime \prime}}\left(L_{\beta}\right)\right)$ and $H_{k}\left(C_{\varepsilon^{\prime}}\left(L_{\alpha}\right)\right) \rightarrow H_{k}\left(C_{\varepsilon^{\prime}}\left(L_{\beta}\right)\right)$. If ever these lower and upper bounds happened to be equal for all $\alpha \leq \beta$, then we could conclude that $\left(\left\{\Phi_{\alpha}\right\},\left\{\phi_{\alpha}^{\beta}\right\}\right)$ has the same persistence diagram as the $k$ th persistent homology module of the filtration $\left\{C_{\varepsilon^{\prime}}\left(L_{\alpha}\right)\right\}_{\alpha \in \mathbb{R}}$, which by Lemmas 1 and 2 is $c \varepsilon^{\prime}$-close to the $k$ th persistence diagram of $f$. However, in full generality the lower and upper bounds may differ.

On the other hand, Lemma 1 tells us that $\left(\left\{\Psi_{\alpha}\right\},\left\{\psi_{\alpha}^{\beta}\right\}\right)$ is related to the homology of the $\varepsilon-, \varepsilon^{\prime}$, and $\varepsilon^{\prime \prime}$-offsets of $L_{\alpha}$ through the following sequence of homomorphisms induced by inclusions: for all $\alpha, \beta$ such that $\beta-\alpha \geq c\left(\varepsilon+\varepsilon^{\prime \prime}\right)$,

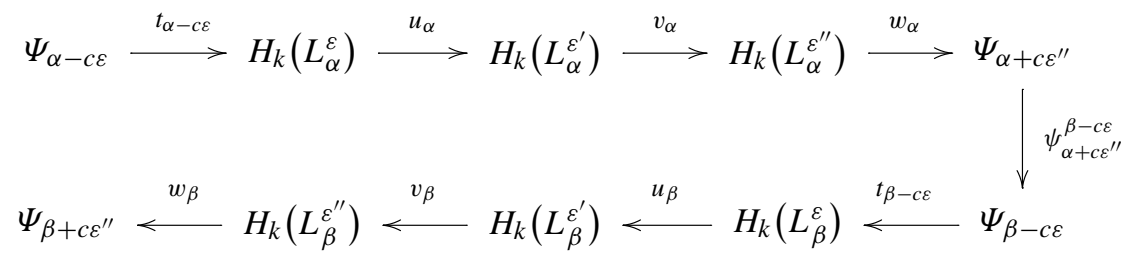

In order to relate (5) to (6), for all $\alpha \in \mathbb{R}$, we consider the isomorphisms $h_{\alpha}$ : $H_{k}\left(C_{\varepsilon}\left(L_{\alpha}\right)\right) \rightarrow H_{k}\left(L_{\alpha}^{\varepsilon}\right), h_{\alpha}^{\prime}: H_{k}\left(C_{\varepsilon^{\prime}}\left(L_{\alpha}\right)\right) \rightarrow H_{k}\left(L_{\alpha}^{\varepsilon^{\prime}}\right)$, and $h_{\alpha}^{\prime \prime}: H_{k}\left(C_{\varepsilon^{\prime \prime}}\left(L_{\alpha}\right)\right) \rightarrow$ $H_{k}\left(L_{\alpha}^{\varepsilon^{\prime \prime}}\right)$ provided by Lemma 2 and well defined since $\varepsilon \leq \varepsilon^{\prime} \leq \varepsilon^{\prime \prime}<\varrho(\mathbb{X})$. Through the diagram of (4), these isomorphisms relate (5) to (6) and thereby draw a connection between the persistence modules $\left(\left\{\Phi_{\alpha}\right\},\left\{\phi_{\alpha}^{\beta}\right\}\right)$ and $\left(\left\{\Psi_{\alpha}\right\},\left\{\psi_{\alpha}^{\beta}\right\}\right)$. Note however that the diagram obtained by combining (4), (5), and (6) may not fully commute: for instance, there is no particular reason why the linear map $m_{\alpha}^{\beta}$ should be identical to $d_{\beta} \circ b_{\beta} \circ a_{\beta} \circ h_{\beta}^{-1} \circ t_{\beta-c \varepsilon} \circ \psi_{\alpha+c \varepsilon^{\prime \prime}}^{\beta-c \varepsilon} \circ w_{\alpha} \circ h_{\alpha}^{\prime \prime} \circ e_{\alpha}$. Nevertheless, the subdiagram of (5) commutes for all $\alpha \leq \beta$, because it is induced by inclusions. Furthermore, 
Lemma 2 ensures that the following subdiagrams (where homomorphisms $l_{\alpha}^{\prime \beta}$ and $n_{\alpha}^{\prime}{ }_{\alpha}$ are induced by inclusions) also commute for all $\alpha \leq \beta$ :

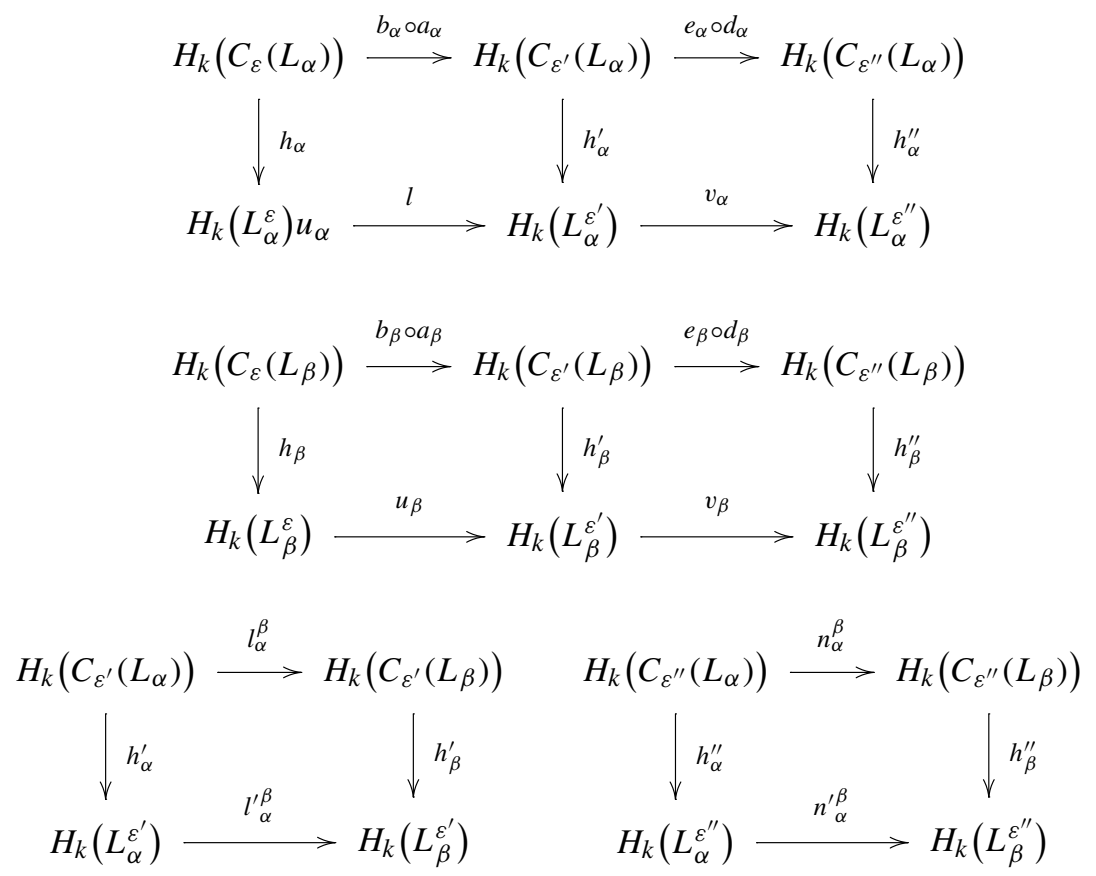

For all $\alpha \in \mathbb{R}$, let $\mu_{\alpha}: \Phi_{\alpha} \rightarrow \Psi_{\alpha+c \varepsilon^{\prime \prime}}$ be the restriction of the map $w_{\alpha} \circ h_{\alpha}^{\prime \prime} \circ e_{\alpha}$ to the subspace $\Phi_{\alpha}=\operatorname{im} d_{\alpha} \circ b_{\alpha} \subseteq H_{k}\left(G_{\alpha}^{\prime}\right)$. Symmetrically, let $v_{\alpha-c \varepsilon}: \Psi_{\alpha-c \varepsilon} \rightarrow \Phi_{\alpha}$ be the map $d_{\alpha} \circ b_{\alpha} \circ a_{\alpha} \circ h_{\alpha}^{-1} \circ t_{\alpha-c \varepsilon}$. Its image is indeed included in the subspace $\Phi_{\alpha}=\operatorname{im} d_{\alpha} \circ b_{\alpha} \subseteq H_{k}\left(G_{\alpha}^{\prime}\right)$. To prove that the persistence modules $\left(\left\{\Phi_{\alpha}\right\},\left\{\phi_{\alpha}^{\beta}\right\}\right)$ and $\left(\left\{\Psi_{\alpha}\right\},\left\{\psi_{\alpha}^{\beta}\right\}\right)$ are $c \varepsilon^{\prime \prime}$-interleaved, it suffices to show that the families of homomorphisms $\left\{\mu_{\alpha}\right\}$ and $\left\{v_{\alpha}\right\}$ make the diagrams of Definition 2 commute for all values $\alpha \leq \beta \in \mathbb{R}$.

We begin with the trapezoids of (2). Consider the map $\mu_{\beta} \circ m_{\alpha}^{\beta} \circ v_{\alpha-c \varepsilon}$. Replacing $\mu_{\beta}$ and $v_{\alpha-c \varepsilon}$ by their definitions, we get $w_{\beta} \circ h_{\beta}^{\prime \prime} \circ\left(e_{\beta} \circ m_{\alpha}^{\beta}\right) \circ d_{\alpha} \circ b_{\alpha} \circ a_{\alpha} \circ h_{\alpha}^{-1} \circ$ $t_{\alpha-c \varepsilon}$, which by commutativity of (5) is equal to $w_{\beta} \circ h_{\beta}^{\prime \prime} \circ\left(n_{\alpha}^{\beta} \circ e_{\alpha}\right) \circ d_{\alpha} \circ b_{\alpha} \circ a_{\alpha} \circ$ $h_{\alpha}^{-1} \circ t_{\alpha-c \varepsilon}$. Now, by commutativity of (7), we have $e_{\alpha} \circ d_{\alpha} \circ b_{\alpha} \circ a_{\alpha} \circ h_{\alpha}^{-1}=h_{\alpha}^{\prime \prime}-1$ 。 $v_{\alpha} \circ u_{\alpha}$, therefore $\mu_{\beta} \circ m_{\alpha}^{\beta} \circ v_{\alpha-c \varepsilon}$ is equal to $w_{\beta} \circ\left(h_{\beta}^{\prime \prime} \circ n_{\alpha}^{\beta} \circ{h^{\prime \prime}}_{\alpha}^{-1}\right) \circ v_{\alpha} \circ u_{\alpha} \circ t_{\alpha-c \varepsilon}$, which by commutativity of the rightmost diagram of (9) is equal to $w_{\beta} \circ n_{\alpha}^{\prime \beta} \circ v_{\alpha} \circ$ $u_{\alpha} \circ t_{\alpha-c \varepsilon}$, which is precisely $\psi_{\alpha-c \varepsilon}^{\beta+c \varepsilon^{\prime \prime}}$.

Consider now the map $v_{\beta-c \varepsilon} \circ \psi_{\alpha+c \varepsilon^{\prime \prime}}^{\beta-c \varepsilon} \circ \mu_{\alpha}$. Since by definition we have $\Phi_{\alpha}=$ $\operatorname{im} d_{\alpha} \circ b_{\alpha} \subseteq \operatorname{im} d_{\alpha}$, the fact that $\nu_{\beta-c \varepsilon} \circ \psi_{\alpha+c \varepsilon^{\prime \prime}}^{\beta-c \varepsilon} \circ \mu_{\alpha}$ coincides with $m_{\alpha}^{\beta}$ over $\Phi_{\alpha}$ is a direct consequence of the fact that the map $v_{\beta-c \varepsilon} \circ \psi_{\alpha+c \varepsilon^{\prime \prime}}^{\beta-c \varepsilon} \circ \mu_{\alpha} \circ d_{\alpha}$ coincides with $m_{\alpha}^{\beta} \circ d_{\alpha}$ over $H_{k}\left(C_{\mathcal{E}^{\prime}}\left(L_{\alpha}\right)\right)$, which we will now prove. Replacing $\mu_{\alpha}$ and $v_{\beta-c \varepsilon}$ by 
their definitions, we get $d_{\beta} \circ\left(b_{\beta} \circ a_{\beta} \circ h_{\beta}^{-1}\right) \circ t_{\beta-c \varepsilon} \circ \psi_{\alpha+c \varepsilon^{\prime \prime}}^{\beta-c \varepsilon} \circ w_{\alpha} \circ\left(h^{\prime \prime}{ }_{\alpha} \circ e_{\alpha} \circ d_{\alpha}\right)$, which by commutativity of (7) and (8) is equal to $d_{\beta} \circ h_{\beta}^{\prime-1} \circ u_{\beta} \circ t_{\beta-c \varepsilon} \circ \psi_{\alpha+c \varepsilon^{\prime \prime}}^{\beta-c \varepsilon}$ $w_{\alpha} \circ v_{\alpha} \circ h_{\alpha}^{\prime}$. Now, observe that $u_{\beta} \circ t_{\beta-c \varepsilon} \circ \psi_{\alpha+c \varepsilon^{\prime \prime}}^{\beta-c \varepsilon} \circ w_{\alpha} \circ v_{\alpha}$ is nothing but the homomorphism $l_{\alpha}^{\prime \beta}$ induced by the inclusion $L_{\alpha}^{\varepsilon^{\prime}} \hookrightarrow L_{\beta}^{\varepsilon^{\prime}}$. Therefore, we have $v_{\beta-c \varepsilon} \circ$ $\psi_{\alpha+c \varepsilon^{\prime \prime}}^{\beta-c \varepsilon} \circ \mu_{\alpha} \circ d_{\alpha}=d_{\beta} \circ\left({h^{\prime}}_{\beta}^{-1} \circ l^{\prime}{ }_{\alpha}^{\beta} \circ h^{\prime}{ }_{\alpha}\right)$, which is equal to $d_{\beta} \circ l_{\alpha}^{\beta}$ by commutativity of the leftmost diagram of (9). Finally, we have $d_{\beta} \circ l_{\alpha}^{\beta}=m_{\alpha}^{\beta} \circ d_{\alpha}$ by commutativity of (5). Thus, $v_{\beta-c \varepsilon} \circ \psi_{\alpha+c \varepsilon^{\prime \prime}}^{\beta-c \varepsilon} \circ \mu_{\alpha}$ coincides with $m_{\alpha}^{\beta}$ over $\Phi_{\alpha}$.

It follows from the two paragraphs above that the trapezoids of (2) commute for all values $\alpha \leq \beta \in \mathbb{R}$. Before analyzing the case of the parallelograms of (3), let us point out that Lemma 2 also guarantees the commutativity of the following subdiagram:

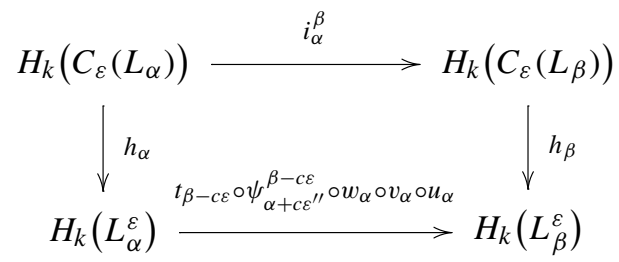

In addition, the following diagram commutes since all homomorphisms are induced by inclusions:

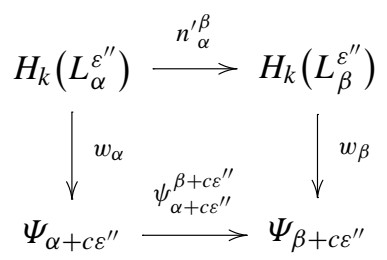

Consider the map $\mu_{\beta} \circ m_{\alpha}^{\beta}$. Replacing $\mu_{\beta}$ by its definition, we obtain $w_{\beta} \circ h_{\beta}^{\prime \prime} \circ\left(e_{\beta} \circ\right.$ $\left.m_{\alpha}^{\beta}\right)$, which by commutativity of (5) is equal to $w_{\beta} \circ\left(h_{\beta}^{\prime \prime} \circ n_{\alpha}^{\beta}\right) \circ e_{\alpha}$. This map is the same as $\left(w_{\beta} \circ{n^{\prime}}_{\alpha}^{\beta}\right) \circ h_{\alpha}^{\prime \prime} \circ e_{\alpha}$, by commutativity of the rightmost diagram of (9). Finally, the commutativity of (11) implies equality with $\psi_{\alpha+c \varepsilon^{\prime \prime}}^{\beta+c \varepsilon^{\prime \prime}} \circ\left(w_{\alpha} \circ h_{\alpha}^{\prime \prime} \circ e_{\alpha}\right)=$ $\psi_{\alpha+c \varepsilon^{\prime \prime}}^{\beta+c \varepsilon^{\prime \prime}} \circ \mu_{\alpha}$.

Consider now the map $m_{\alpha}^{\beta} \circ v_{\alpha-c \varepsilon}$. Replacing $v_{\alpha}$ by its definition, we obtain $\left(m_{\alpha}^{\beta} \circ\right.$ $\left.d_{\alpha} \circ b_{\alpha} \circ a_{\alpha}\right) \circ h_{\alpha}^{-1} \circ t_{\alpha-c \varepsilon}$, which by commutativity of (5) is equal to $d_{\beta} \circ b_{\beta} \circ a_{\beta} \circ$ $\left(i_{\alpha}^{\beta} \circ h_{\alpha}^{-1}\right) \circ t_{\alpha-c \varepsilon}$. By commutativity of (10), this map coincides with $d_{\beta} \circ b_{\beta} \circ a_{\beta} \circ$ $h_{\beta}^{-1} \circ t_{\beta-c \varepsilon} \circ\left(\psi_{\alpha+c \varepsilon^{\prime \prime}}^{\beta-c \varepsilon} \circ w_{\alpha} \circ v_{\alpha} \circ u_{\alpha} \circ t_{\alpha-c \varepsilon}\right)$, which is equal to $\left(d_{\beta} \circ b_{\beta} \circ a_{\beta} \circ h_{\beta}^{-1} \circ\right.$ $\left.t_{\beta-c \varepsilon}\right) \circ \psi_{\alpha-c \varepsilon}^{\beta-c \varepsilon}=v_{\beta-c \varepsilon} \circ \psi_{\alpha-c \varepsilon}^{\beta-c \varepsilon}$ since the homomorphisms in (6) are induced by inclusions.

It follows from the two paragraphs above that the parallelograms of (3) commute for all values $\alpha \leq \beta \in \mathbb{R}$. This concludes the proof that the persistence modules $\left(\left\{\Phi_{\alpha}\right\},\left\{\phi_{\alpha}^{\beta}\right\}\right)$ and $\left(\left\{\Psi_{\alpha}\right\},\left\{\psi_{\alpha}^{\beta}\right\}\right)$ are $c \varepsilon^{\prime \prime}$-interleaved. 


\subsection{Stability with Respect to Noise}

The guarantees provided by Theorem 2 hold as far as exact geodesic distances and function values are used in the construction of the Rips complexes. In practice however, function values are often obtained from physical measurements with inherent noise, while geodesic distances are not known in advance and have to be estimated through some neighborhood graph distance. We claim that our analysis is generic enough to handle these practical situations.

Consider first the case where function values are noisy. More precisely, given a geodesic $\varepsilon$-sample $L$ of some Riemannian manifold $\mathbb{X}$, and a $c$-Lipschitz function $f$ : $\mathbb{X} \rightarrow \mathbb{R}$, assume that the data points $p \in L$ are assigned values $\tilde{f}(p)$ that are different from $f(p)$, and let $\zeta=\max _{p \in L}|\tilde{f}(p)-f(p)|$. Note that the map $\tilde{f}: L \rightarrow \mathbb{R}$ itself need not be Lipschitz continuous. For convenience, for all $\alpha \in \mathbb{R}$, we introduce the set $\tilde{L}_{\alpha}$ of points of $L$ whose $\tilde{f}$-values are at most $\alpha$. Note that $\tilde{L}_{\alpha}$ may neither contain nor be contained in $L_{\alpha}$ in general. However, we have $\tilde{L}_{\alpha} \subseteq L_{\alpha+\zeta}$, which, plugged into the proof of Lemma 1, yields the following variant of that result:

Lemma 6 For all $\delta \geq \varepsilon$, the sublevel-sets filtration of $f$ is $(c \delta+\zeta)$-interleaved with $\left\{\tilde{L}_{\alpha}^{\delta}\right\}_{\alpha \in \mathbb{R}}$.

The rest of the analysis of Sects. 3.1 and 3.2 carries through, with $L_{\alpha}$ replaced by $\tilde{L}_{\alpha}$ for all $\alpha \in \mathbb{R}$ and $c \varepsilon$ and $c \varepsilon^{\prime \prime}$ replaced respectively by $c \varepsilon+\zeta$ and $c \varepsilon^{\prime \prime}+\zeta$ in (6) and in the rest of the proof of Lemma 5. We thus obtain the following new bounds:

Theorem 3 Let $\mathbb{X}, f, L$ be as in Theorem 2. Assume that the values of $f$ at the points of $L$ are known within a precision of $\zeta$. Then, for any $\delta \in\left[2 \varepsilon, \frac{1}{2} \varrho(\mathbb{X})\right)$ and any $k \in \mathbb{N}$, the kth persistent homology modules of $f$ and of the nested pair of filtrations $\left\{R_{\delta}\left(\tilde{L}_{\alpha}\right) \hookrightarrow R_{2 \delta}\left(\tilde{L}_{\alpha}\right)\right\}_{\alpha \in \mathbb{R}}$ are $(2 c \delta+\zeta)$-interleaved. Therefore, the bottleneck distance between their persistence diagrams is at most $2 c \delta+\zeta$, by Theorem 1 .

Consider now the case where geodesic distances are noisy. Specifically, assume that the geodesic distance $\mathrm{d}_{\mathbb{X}}$ is replaced by the distance $\mathrm{d}_{G}$ in some neighborhood graph $G$ built on top of the point cloud $L$. This graph can be either weighted of unweighted, depending on the application. For instance, in unsupervised learning the edges of $G$ are often weighted by the Euclidean distances between their vertices [32], while in sensor networks edges are usually unweighted because retrieving the exact geographic locations of the sensor nodes can be difficult—see e.g., [33, §4.4]. Generally speaking, weighted graphs provide better approximations of geodesic distances, but their construction requires to have additional information at hand, such as extrinsic distances between the data points. We therefore focus on unweighted graphs, which correspond to the most general case. In order to make theoretical claims, we assume that $G$ is a $\mu$-disk graph, ${ }^{6}$ that is, a pair of data points form an edge in $G$ if and only if their geodesic distance is less than $\mu$. Assuming that the input point cloud

\footnotetext{
${ }^{6}$ Our analysis holds also for quasi $\mu$-disk graphs, modulo a slight degradation of the approximation bounds.
} 
$L$ is a geodesic $\varepsilon$-sample of some Riemannian manifold $\mathbb{X}$, we will use the following bounds on the graph distance [22, Lemma 6.1]:

$$
\forall p, q \in L, \quad \frac{\mathrm{d}_{\mathbb{X}}(p, q)}{\mu} \leq \mathrm{d}_{G}(p, q) \leq 1+\lambda \frac{\mathrm{d}_{\mathbb{X}}(p, q)}{\mu}, \quad \text { where } \lambda=1+4 \frac{\varepsilon}{\mu} .
$$

The two Rips-based filtrations introduced at the beginning of Sect. 3 are now defined with respect to $\mathrm{d}_{G}$, and no longer with respect to $\mathrm{d}_{\mathbb{X}}$. To emphasize this aspect, we denote them respectively by $\left\{R_{\delta}^{G}\left(L_{\alpha}\right)\right\}_{\alpha \in \mathbb{R}}$ and $\left\{R_{\delta^{\prime}}^{G}\left(L_{\alpha}\right)\right\}_{\alpha \in \mathbb{R}}$. In Theorem 2 we set $\delta^{\prime}=2 \delta$ because geodesic distances were exact. We will now show that noise in geodesic distances can be handled by taking a slightly larger $\delta^{\prime}$. We first relate $\left\{R_{\delta}^{G}\left(L_{\alpha}\right)\right\}_{\alpha \in \mathbb{R}}$ and $\left\{R_{\delta^{\prime}}^{G}\left(L_{\alpha}\right)\right\}_{\alpha \in \mathbb{R}}$ to Čech filtrations defined with respect to $\mathrm{d}_{\mathbb{X}}$ :

Lemma 7 Let $\lambda=1+4 \frac{\varepsilon}{\mu}$ be as in (12). Assume that $\delta \geq 1+2 \lambda \frac{\varepsilon}{\mu}$, $\varepsilon^{\prime} \geq \mu \delta, \delta^{\prime} \geq$ $1+2 \lambda \frac{\varepsilon^{\prime}}{\mu}$, and $\varepsilon^{\prime \prime} \geq \mu \delta^{\prime}$. Then, for all $\alpha \in \mathbb{R}, \quad C_{\varepsilon}\left(L_{\alpha}\right) \subseteq R_{\delta}^{G}\left(L_{\alpha}\right) \subseteq C_{\varepsilon^{\prime}}\left(L_{\alpha}\right) \subseteq$ $R_{\delta^{\prime}}^{G}\left(L_{\alpha}\right) \subseteq C_{\varepsilon^{\prime \prime}}\left(L_{\alpha}\right)$.

Proof Let us prove that $R_{\xi}^{G}\left(L_{\alpha}\right) \subseteq C_{\mu \xi}\left(L_{\alpha}\right)$ and $C_{\xi}\left(L_{\alpha}\right) \subseteq R_{1+2 \lambda \xi / \mu}^{G}\left(L_{\alpha}\right)$ for any arbitrary value $\xi \geq 0$. The lemma will then follow by letting $\xi$ be consecutively equal to $\varepsilon, \delta, \varepsilon^{\prime}$, and $\delta^{\prime}$.

Consider first a simplex $\left\{p_{0}, \ldots, p_{l}\right\}$ of $R_{\xi}^{G}\left(L_{\alpha}\right)$. Equation (12) implies that $\mathrm{d}_{\mathbb{X}}\left(p_{0}, p_{i}\right) \leq \mu \mathrm{d}_{G}\left(p_{0}, p_{i}\right)<\mu \xi$ for all $i \in\{0, \ldots, l\}$. This means that the open geodesic balls of same radius $\mu \xi$ about the points $p_{i}$ have $p_{0}$ in their common intersection, which is therefore nonempty. As a consequence, the simplex belongs to $C_{\mu \xi}\left(L_{\alpha}\right)$. Consider now a simplex $\left\{p_{0}, \ldots, p_{l}\right\}$ of $C_{\xi}\left(L_{\alpha}\right)$. The open geodesic balls of same radius $\xi$ about the points $p_{i}$ have a nonempty common intersection, and therefore the pairwise geodesic distances between the points are less than $2 \xi$. It follows then from (12) that the diameter of the simplex in the graph distance is at most $1+2 \lambda \frac{\xi}{\mu}$. Thus, the simplex belongs to $R_{1+2 \lambda \xi / \mu}^{G}\left(L_{\alpha}\right)$.

Letting $G_{\alpha}=R_{\delta}^{G}\left(L_{\alpha}\right)$ and $G_{\alpha}^{\prime}=R_{\delta^{\prime}}^{G}\left(L_{\alpha}\right)$, where $\delta, \delta^{\prime}$ and $\varepsilon \leq \varepsilon^{\prime} \leq \varepsilon^{\prime \prime}$ satisfy the conditions of Lemma 7, we can now apply Lemma 5 to get the following guarantee:

Theorem 4 Let $\mathbb{X}, f, L$ be as in Theorem 2. Assume that the geodesic distance $\mathrm{d}_{\mathbb{X}}$ is replaced by the graph distance $\mathrm{d}_{G}$ in the unweighted $\mu$-disk graph $G$ built on top of $L$. Let $\lambda=1+4 \frac{\varepsilon}{\mu}$. Then, for any $\delta \geq 1+2 \lambda \frac{\varepsilon}{\mu}$, any $\delta^{\prime} \in\left[1+2 \lambda \delta, \frac{1}{\mu} \varrho(\mathbb{X})\right)$, and any $k \in \mathbb{N}$, the kth persistent homology modules of $f$ and of the nested pair of filtrations $\left\{R_{\delta}^{G}\left(L_{\alpha}\right) \hookrightarrow R_{\delta^{\prime}}^{G}\left(L_{\alpha}\right)\right\}_{\alpha \in \mathbb{R}}$ are $c \mu \delta^{\prime}$-interleaved. Therefore, the bottleneck distance between their persistence diagrams is at most $c \mu \delta^{\prime}$, by Theorem 1.

This result provides sufficient conditions on parameters $\delta, \delta^{\prime}$ for the analysis of the previous sections to hold in the case where geodesic distances are not exact. Note that simple expressions can be derived for $\delta$ and $\delta^{\prime}$, which can be later used in our algorithms. For instance, if we assume that $\mu \geq 4 \varepsilon$, then $\lambda \leq 2$, and therefore we can choose $\delta=2$ and $\delta^{\prime}=9$. Then, the conclusion of Theorem 4 holds, provided that $\delta^{\prime}$ 
is less than $\frac{1}{\mu} \varrho(\mathbb{X})$, from which we derive the following condition on the sampling density $\varepsilon$ and communication radius $\mu: 4 \varepsilon \leq \mu<\frac{1}{9} \varrho(\mathbb{X})$.

Note finally that Theorems 3 and 4 can also be combined to obtain guarantees in scenarios where both function values and pairwise distances are approximate.

\section{Algorithms}

Section 4.1 presents the core algorithm, which derives from the structural results of Sect. 3. The subsequent sections introduce two improvements to the algorithm: the first one deals with time-varying functions (Sect. 4.2), and the second one extracts additional spatial information (Sect. 4.3).

\subsection{Core Algorithm}

The algorithm takes as input an $n$-dimensional vector $v$, an $n \times n$ distance matrix $D$, and a parameter $\delta \geq 0$. The $i$ th entry of $v$ stands for the function value at the $i$ th point of the data set, while the entries $D_{i, j}=D_{j, i}$ give the distance between points $i$ and $j$. No geographic coordinates are to be provided, so that the algorithm can virtually be applied in any arbitrary metric space. For clarity of exposition, we assume that the entries of $v$ are sorted, that is, $v_{1} \leq v_{2} \leq \cdots \leq v_{n}$. They are not in our implementation. The algorithm proceeds in two steps:

1. It builds two families of Rips complexes, $R_{\delta}(\{1\}) \subseteq R_{\delta}(\{1,2\}) \subseteq \cdots \subseteq R_{\delta}(\{1,2$, $\ldots, n\})$ and $R_{2 \delta}(\{1\}) \subseteq R_{2 \delta}(\{1,2\}) \subseteq \cdots \subseteq R_{2 \delta}(\{1,2, \ldots, n\})$. The $i$ th complex in each family is computed from the submatrix of $D$ spanned by the rows and columns of indices $1, \ldots, i$. The time of appearance of its simplices that are not in the $(i-1)$ th complex is set to $v_{i}$.

2. For $k$ ranging from zero to the dimension of the complexes, it computes the $k$ th persistence diagram of the nested pair of filtrations $\left\{R_{\delta}(\{1, \ldots, i\}) \hookrightarrow\right.$ $\left.R_{2 \delta}(\{1, \ldots, i\})\right\}_{1 \leq i \leq n}$.

Upon termination, the algorithm returns the persistence diagrams computed at step 2. The quality of this output is guaranteed by the structural results of Sect. 3, under sufficient sampling density and in the absence of noise. Observe indeed that the filtrations built at step 1 are the same as the ones considered in Theorem 2, which therefore provides the following theoretical guarantee:

Theorem 5 If the data points form a geodesic $\varepsilon$-sample of some Riemannian manifold $\mathbb{X}$ with $\varepsilon<\frac{1}{4} \varrho(\mathbb{X})$ and if the input distance matrix $D$ gives the exact geodesic distances between the data points, then, for any input $\delta \in\left[2 \varepsilon, \frac{1}{2} \varrho(\mathbb{X})\right)$ and any $c$ Lipschitz function $f: \mathbb{X} \rightarrow \mathbb{R}$ whose values at the data points are given exactly by the input vector $v$, the kth persistence diagram output by the algorithm lies at bottleneck distance at most $2 c \delta$ of the kth persistence diagram of $f$.

Note that the output of the algorithm also gives the homology groups of the underlying space $\mathbb{X}$. Indeed, $H_{k}(\mathbb{X})$ is isomorphic to the linear span of the $k$-dimensional 
homological features that are infinitely persistent in the $k$ th persistence diagram of $f$. Now, by Theorem 5, the bottleneck distance between the diagram of $f$ and the one computed at step 2 of the algorithm is finite, and therefore the infinitely persistent homological features in both diagrams are in bijection.

One drawback of our approach is that it is not parameter-free, which makes its behavior dependent on the choice of the input parameter $\delta$. In some sense, this parameter controls the scale at which the algorithm will process the data. The issue of finding the right scale is ubiquitous in geometric data analysis, and several solutions based on the idea of persistence have been proposed. We suggest to consider a whole range of values of $\delta$ between zero and infinity (or any sufficiently large value). For each value in this range, ${ }^{7}$ we apply the algorithm and report the infinitely persistent homological features in the output persistence diagrams, which supposedly coincide with the ones of the underlying space $\mathbb{X}$, according to Theorem 5 . Then, following [23] and subsequent work, we claim that relevant ranges of scales can be identified as ranges of values of $\delta$ over which the numbers of infinitely persistent homological features in all the diagrams are stable.

Finally, note that Theorems 3 and 4 provide theoretical guarantees similar to the ones of Theorem 5 in cases where the input vector $v$ of function values or the input distance matrix $D$ is noisy, modulo some slight modifications in the choice of parameters of the algorithm, as explained in Sect. 3.3.

Implementation and Complexity The running time of the algorithm can be bounded in terms of the size of the data structure, provided that a careful implementation is built. In our case, the two families of complexes introduced at step 1 are built simultaneously as filtrations of the largest of the Rips complexes, $R_{2 \delta}(\{1, \ldots, n\})$, which by definition contains all the other complexes of the two families. As emphasized in [12], the simplices of $R_{2 \delta}(\{1, \ldots, n\})$ are in bijection with the cliques of its 1-skeleton graph. Therefore, we first build this graph in $O\left(n^{2}\right)$ time by comparing the entries of the matrix $D$ with the threshold $2 \delta$. Then, we construct the simplices of $R_{2 \delta}(\{1, \ldots, n\})$ iteratively, by increasing dimension. First, all vertices are created. Then, for each simplex $\left\{i_{0}, \ldots, i_{k}\right\}$ created, we look at its 1 -ring neighborhood in the graph, and for each vertex $i_{l}$ in this neighborhood, we check whether $\left\{i_{0}, \ldots, i_{k}, i_{l}\right\}$ forms a clique. If so, then this new simplex is created, and its diameter $\max _{1 \leq r<s \leq l} D_{r, s}$ and appearance time $\max _{1 \leq r \leq l} v_{j_{r}}$ are stored. The time spent checking whether we have a clique and computing the new diameter and appearance time from the ones of the original simplex $\left\{i_{0}, \ldots, i_{k}\right\}$ is $O(k)$, while the size of the 1-ring neighborhood is $O(n)$. Thus, the total time spent building the complex is $O(n d N)$, where $d$ is the dimension of the complex, and $N$ is its total number of simplices. Then, within $O(N \log N)$ time, we order the created simplices according to their appearance times, to build the filtration of parameter $2 \delta$. As for the filtration of parameter $\delta$, observe that each of its simplices must appear in both filtrations at the same time. Therefore, we can build the filtration of parameter $\delta$ in $O(N)$ time by scanning through the sorted list of simplices in the filtration of parameter $2 \delta$ and

\footnotetext{
${ }^{7}$ In fact, we only have to consider the finitely many values of $\delta$ at which the combinatorial structures of the Rips complexes change.
} 
reporting the simplices that have diameter at most $\delta$. Finally, we perform step 2 by running the algorithm of [14] on our two filtrations. This variant of the standard persistence algorithm has the same worst-case running time of $O\left(N^{3}\right)$.

Theorem 6 The total running time of the algorithm is $O\left(n d N+N \log N+N^{3}\right)$, where $d$ is the dimension of $R_{2 \delta}(\{1, \ldots, n\})$, and $N$ is its total number of simplices.

Step 2 is clearly the pacing phase of our method. However, it is reported in [35] that, although the worst-case running time of the persistence algorithm is $O\left(N^{3}\right)$, in most practical cases it has an almost-linear behavior. Thus, the running time of our method is likely to be $O(n d N+N \log N)$ in practice.

Note also that $R_{2 \delta}(\{1, \ldots, n\})$ could potentially span the full $(n-1)$-simplex and therefore have as many as $2^{n}$ simplices. However, there are important cases where the size of the complex remains bounded. For instance, when the data points are uniformly sampled along an $m$-dimensional Riemannian manifold, a packing argument detailed in [12] shows that the size of the complex is at most $2^{2^{m}} n$ and that it even reduces to $2^{O\left(m^{2}\right)} n$ if a reasonable upper bound on $m$ is known. This reduces the running time of the algorithm to $2^{O\left(m^{2}\right)} n^{3}$ and thereby makes the approach tractable when the data points sample uniformly some low-dimensional manifold, possibly embedded in a high-dimensional space. Sampling uniformity can be achieved in practice by a landmarking strategy [23].

\subsection{Time-varying Functions}

It is commonplace in sensor networks and related areas that the functions under study vary with time. In monitoring applications, for instance, one wants to get a high-level description of the distribution of some intensive quantity like temperature or humidity over a fixed domain. Such quantities vary typically on a day scale, and a natural goal is to be able to maintain accurate approximations to their persistence diagrams under such variations.

We model the problem as follows: given a finite point cloud $L=\left\{x_{1}, \ldots, x_{n}\right\}$ that is a geodesic $\varepsilon$-sample of some fixed Riemannian manifold $\mathbb{X}$, we want to maintain accurate approximations to the persistence diagrams of some time-varying function $f_{t}: \mathbb{X} \rightarrow \mathbb{R}$ whose values are known only at the points of $L$ and at a finite number of instants $t_{0} \leq t_{1} \leq \cdots \leq t_{k}$. We assume $f_{t_{i}}$ to be $c$-Lipschitz for all $i$ and some fixed constant $c$. Thanks to this assumption, Theorem 5 provides us with theoretical guarantees regarding the quality of the output persistence diagrams at every instant $t_{i}$. The dynamic version of the algorithm works as follows:

- It performs an initialization step at time $t_{0}$, where it simply applies the core algorithm as in the static setting. The filtrations of parameters $\delta$ and $2 \delta$ are stored as two arrays of simplices, sorted according to their times of appearance, which are derived from the values of $f_{t_{0}}$ at the vertices.

- At every subsequent instant $t_{j}$ we need to update the two filtrations and then to recompute their $k$ th persistence diagram for all values $k$ between zero and their dimension. In fact, since $\mathbb{X}$ and $L$ remain fixed throughout the process, the distance matrix $D$ does not change, and therefore the Rips complexes $R_{\delta}(L)$ and $R_{2 \delta}(L)$ 
remain the same. Thus, updating the filtrations boils down to resorting their simplices according to the new appearance times induced by $f_{t_{j}}$. Computing the new appearance times is done by scanning through the filtrations and, for each simplex, finding the vertex of maximal $f_{t_{j}}$-value. Then, resorting the simplices of each filtration is done in-place in the array of the filtration using insertion sort. The reason for using this particular sorting algorithm is that it decomposes the permutation on the simplices into a sequence of inversions. ${ }^{8}$ This sequence is then provided as input to the vineyards ${ }^{9}$ variant of the persistence algorithm [15], which uses this information to update the $k$ th persistence diagram for all values $k$ at once.

The time complexity of the initialization stage is the same as the one of the static algorithm, namely $O\left(N^{3}\right)$, where $N$ is the total number of simplices of $R_{2 \delta}(L)$. Then, at every subsequent instant $t_{j}$, the time spent updating the appearance times is $O(d N)$, where $d$ is the dimension of $R_{2 \delta}(L)$. Consider now the permutation $\pi_{j}$ on the simplices induced by the change from function $f_{t_{j-1}}$ to function $f_{t_{j}}$. A key feature of insertion sort is that it decomposes $\pi_{j}$ into a minimal sequence of inversions, of size $\left|\pi_{j}\right|$. Its time complexity is thus $O\left(N+\left|\pi_{j}\right|\right)$. Finally, the vineyards algorithm updates the persistence diagrams in $O(N)$ time per inversion. Hence, the total time spent by our method at instant $t_{j}$ is $O\left(\left(1+d+\left|\pi_{j}\right|\right) N\right)$. Although $d$ is bounded by $\log N$, in the worst case, $\left|\pi_{j}\right|$ can be up to $\Theta\left(N^{2}\right)$, thereby raising the complexity to $\Theta\left(N^{3}\right)$, which is no better than if the filtrations and persistence diagrams were recomputed entirely at time $t_{j}$. However, this is a worst-case analysis, and in many practical situations, $\left|\pi_{j}\right|$ is likely to be small. If, for instance, the values $f_{t}\left(x_{i}\right)$ at the data points follow polynomial trajectories in time ${ }^{10}$ such that only a constant number (say two) of such trajectories meet at any given time, then between two instants $t_{j-1}$ and $t_{j}$ that are close enough, only two function values $f_{t}\left(x_{i}\right), f_{t}\left(x_{j}\right)$ are permuted. As a consequence, only the stars of $x_{i}, x_{j}$ in $R_{2 \delta}(L)$ are affected by $\pi_{j}$, and therefore we have $\left|\pi_{j}\right|=O\left(d_{v}^{2}\right)$, where $d_{v}$ denotes the size of the largest possible star of a vertex of $R_{2 \delta}(L)$. The update time of our method at $t_{j}$ becomes then $O\left(\left(d+d_{v}^{2}\right) N\right)=O\left(\left(d+d_{v}^{2}\right) d_{v} n\right)$. If the input point cloud uniformly samples some Riemannian manifold of dimension $m$ (known within a constant factor), then we have $d=O(m)$ and $d_{v}=2^{O\left(m^{2}\right)}$, which reduces the update time to $2^{O\left(m^{2}\right)} n$-a quantity that is linear in the size of the input, modulo a constant factor that depends on the intrinsic dimensionality of the data.

Finally, let us mention that, similarly to the standard persistence algorithm, the vineyards algorithm has been observed to run much faster in practice than expected in theory [15]. Typically, the observed running time is constant per simplex inversion. This reduces the update time of our method to $O\left(d+\left|\pi_{j}\right|\right)$ in the general case, and even to a constant $2^{O\left(\mathrm{~m}^{2}\right)}$ in the practical setting described above.

\footnotetext{
${ }^{8} \mathrm{An}$ inversion is a transposition between two simplices that are adjacent in the array.

${ }^{9}$ Originally designed for a single filtration, this algorithm was adapted to our context in Appendix A of [14].

${ }^{10}$ This is the usual assumption in the kinetic data structures framework [24].
} 


\subsection{Extracting Additional Spatial Information}

Assume the domain $\mathbb{X}$ underlying the data to be an $m$-dimensional Riemannian manifold, and the unknown function $f: \mathbb{X} \rightarrow \mathbb{R}$ to be a Morse function, i.e., a smooth function with only nondegenerate critical points. We want to recover the ascending regions (a.k.a. stable manifolds) of the maxima of $f$ and the descending regions (a.k.a. unstable manifolds) of its minima. The ascending region of a maximum $p$ is the set of points of $\mathbb{X}$ that eventually reach $p$ by moving along the flow induced by the gradient vector field of $f$. Symmetrically, the descending region of a minimum $q$ is the set of points that eventually reach $q$ by moving against the gradient flow. These regions share many interesting properties, among which the following ones are of particular interest to us: ascending (resp. descending) regions form pairwise disjoint open cells homeomorphic to $\mathbb{R}^{m}$ that cover $\mathbb{X}$ up to a subset of measure zero. In other words, they can be used as a tool for segmenting the domain $\mathbb{X}$ according to the basins of attraction of the maxima (resp. minima) of $f$. Furthermore, they can be used as the main building block of the Morse-Smale decomposition of $\mathbb{X}$ induced by $f$, since the faces of the complex are obtained as intersections of ascending and descending regions. Note that the ascending regions of $f$ are the descending regions of $-f$, so the problem reduces to finding the descending regions of $f$ from its values at a finite sampling $L$ of $\mathbb{X}$.

As in the previous sections, the geographic locations of the data points are not assumed to be known, and the algorithm uses only the connectivity between the data points in the 1-skeleton graph of the Rips complex $R_{2 \delta}(L)$, called the Rips graph $G$ from now on. For simplicity, we assume that the values of $f$ at the data points are all different. This genericity condition is easily ensured by an infinitesimal perturbation of $f$. At a high level, our method is composed of two phases: first, it approximates the gradient vector field of $f$ at the vertices of $G$ and clusters them according to the (approximate) basins of attraction in the graph $G$; second, it uses the 0th persistence diagram of $f$ to merge the clusters of short lifespans with longer-lasting clusters. The clustering technique used in the first phase is in fact not new, and it has been shown to be quite unstable under small perturbations of the function, both in theory [18] and in practice [34]. The novelty of our approach lies in the way it uses persistence to merge clusters and regain some stability.

In the first phase, we iterate over the vertices of $G$ in the order of their $f$-values. At each vertex $v$, the direction of $-\nabla f$ is approximated by the edge $e$ of $G$ that connects $v$ to a neighbor $u$ minimizing the quantity $\frac{f(u)-f(v)}{|e|}$, where $|e|$ is the length of the edge, computed during the construction of the Rips graph $G$. If no neighbor of $v$ has a lower $f$-value than $v$, then $v$ is a local minimum of $f$ in $G$ and is therefore kept disconnected. Such a vertex $v$ is called a sink. Note that every nonsink vertex $w$ is connected to a proper neighbor in $G$, and by following the approximate direction of $-\nabla f$ in the graph we eventually reach a sink because the value of $f$ decreases strictly along the path followed. We declare this sink as the center of the cluster to which $w$ belongs.

Recall now that the core algorithm (Sect. 4.1) approximates the $k$ th persistence diagram of $f$ via the $k$ th persistent homology module of the nested pair of filtrations

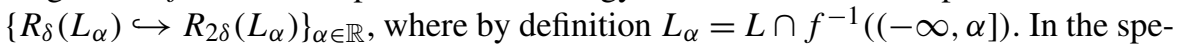
cial case of zero-dimensional homology, however, we know that each vertex appears 
both in $R_{\delta}\left(L_{\alpha}\right)$ and in $R_{2 \delta}\left(L_{\alpha}\right)$ at the same time, and therefore the homomorphism $H_{0}\left(R_{\delta}\left(L_{\alpha}\right)\right) \rightarrow H_{0}\left(R_{2 \delta}\left(L_{\alpha}\right)\right)$ is surjective. As a result, the 0 th persistence diagram of the nested pair of filtrations $\left\{R_{\delta}\left(L_{\alpha}\right) \hookrightarrow R_{2 \delta}\left(L_{\alpha}\right)\right\}_{\alpha \in \mathbb{R}}$ is identical to the 0th persistence diagram of the filtration $\left\{R_{2 \delta}(L)\right\}_{\alpha \in \mathbb{R}}$, which can be computed easily from its 1-skeleton graph $G$ using a variant of the standard union-find data structure, described in [20]. This is what phase two of our algorithm does. The outcome is a set of pairs $(v, e)$, where $v$ is a local minimum of $f$ in the graph $G$, and $e$ is an edge of $G$ that connects the connected component created by $v$ in $G$ to some older connected component. Stated differently, $v$ is a sink, and $e$ is the first edge that connects its cluster to some older cluster of center $u$. If the lifespan ${ }^{11}$ of the cluster of $v$ is shorter than some user-defined threshold $\lambda$, then the algorithm merges the cluster of $v$ into the cluster of $u$.

Our implementation uses only one pass through the graph $G$, during which the approximate gradients at the vertices are computed and the clusters are formed and merged on the fly using the union-find data structure of [20]. Thus, once the Rips graph $G$ is built, the remaining running time is $O\left(|G| A^{-1}(|G|)\right)$, where $|G|$ is the size of $G$, and $A$ is the Ackermann function. In addition, Theorem 5 provides the following theoretical guarantee on the output of the algorithm:

Theorem 7 Assume $L$ to be a geodesic $\varepsilon$-sample of $\mathbb{X}$, with $\varepsilon<\frac{1}{4} \varrho(\mathbb{X})$, and $f$ to be c-Lipschitz. Assume further that there exist two nonnegative values $\mathrm{d}_{2}>\mathrm{d}_{1}+16 c \varepsilon$ such that the 0 th persistence diagram of $f$ has the following well-separated structure: $\mathrm{D}_{0} f=\mathrm{D}_{1} \cup \mathrm{D}_{2}$ with $\max \left\{p_{y}-p_{x}, p \in \mathrm{D}_{1}\right\} \leq \mathrm{d}_{1}$ and $\min \left\{q_{y}-q_{x}, q \in \mathrm{D}_{2}\right\} \geq \mathrm{d}_{2}$. Then, for any Rips parameter $\delta \in\left[2 \varepsilon, \min \left\{\frac{1}{2} \varrho(\mathbb{X}), \frac{\mathrm{d}_{2}-\mathrm{d}_{1}}{8 c}\right\}\right)$ and any threshold $\lambda \in$ $\left(\mathrm{d}_{1}+4 c \delta, \mathrm{d}_{2}-4 c \delta\right)$, the number of clusters computed by our algorithm is equal to the number of basins of attraction of minima of $f$ on $\mathbb{X}$ whose lifespans are at least $\lambda$. Furthermore, there is a pairing between clusters and basins of attraction that modifies the birth times by at most $2 c \delta$.

The well-separatedness of the 0th persistence diagram of $f$ can be interpreted as a signal-to-noise ratio condition: the relevant peaks or valleys of $f$ must be significantly more persistent than the nonrelevant ones, as measured by the difference between their lifespans. Under such a condition, it is possible to threshold the diagrams of $f$ and of the Rips complex $R_{2 \delta}(L)$ so that the remaining finite point sets in both diagrams are in bijection and lie at small bottleneck distance of each other.

In addition to the above stability guarantee, it is desirable to have some guarantees on the locations of our clusters with respect to the basins of attraction of the corresponding minima of $f$. A result in this vein has been obtained in a recent follow-up to this work [10].

${ }^{11}$ Defined as the difference between the times at which $e$ and $v$ appear in the Rips graph $G$. 
Table 1 Timings on an Intel Core 2 Duo T7500 @ $2.20 \mathrm{GHz}$ with 2 GB of RAM. We used the C++ library ANN [1] for the proximity queries involved in the construction of the Rips graph. The clustering phase comprises both steps of the algorithm of Sect. 4.3 , which are performed simultaneously

\begin{tabular}{llrrrrr}
\hline Data set & Dimension & \# vertices & \# edges & Rips graph (s) & Clustering (s) & Total (s) \\
\hline Crater & 2 & 1,048 & 7,095 & 0.01 & 0.00 & 0.01 \\
Torus & 3 & 2,034 & 7,650 & 0.01 & 0.00 & 0.01 \\
Four Gaussians & 2 & 6,354 & 51,946 & 0.07 & 0.02 & 0.09 \\
Hand & 2 & 19,470 & 158,395 & 0.27 & 0.05 & 0.32 \\
Double spiral & 2 & 114,563 & $2,116,035$ & 2.43 & 0.61 & 3.04 \\
Octopus & 3 & 770,196 & $9,540,143$ & 14.56 & 7.11 & 21.67 \\
\hline
\end{tabular}

\section{Applications \& Discussion}

We now illustrate the relevance and generality of our approach through three specific applications. For each application, we describe the context and show some experimentation validation. We also provide timings information in Table 1.

Sensor Networks Our approach was originally designed with the sensor network framework in mind, where physical quantities such as temperature or humidity are measured by a collection of communicating sensors, and where the goal is to answer qualitative queries such as how many significant hot spots are being sensed. Purely geometric approaches cannot be applied in this setting, since geographic location is usually unavailable. Rough pairwise geodesic distances however are available, in the form of graph distances in the communication network. With this data at hand, the algorithms of Sect. 4 can find the number of hot spots, provide an estimation of their prominence and of their size in the network, and track them as the quantity being measured changes.

Because we do not have access to real sensor network data, we test the methods using synthetic examples. In particular, we assume a unit-disk graph model as the communication model and test the effect of different communication radii on the approximation as compared to the persistence we would compute if we had the node locations. Note that under this model, the communication graph is equivalent to a Rips graph, and so we present the results in the next section independently of the sensor network motivation.

Synthetic Examples We test the validity of our theoretical guarantees by comparing the persistence computed using a triangulation and one computed using the Rips complex. We considered three functions on the unit-square: a mixture of 4 Gaussians (Fig. 2(a)), its opposite, and a function with the shape of a crater (Fig. 3(a)). For this experiment, we first constructed a uniform 0.05-sampling of the unit square and sampled the three functions. As ground-truth, we take the piecewise linear function on the Delaunay triangulation of the points.

We compute the persistence diagrams of the three functions on the triangulations and compare them to the diagrams computed using the Rips complex. In Figs. 2(b-d) and $3(b-c)$, we show the persistence diagrams for the piecewise linear function (in 


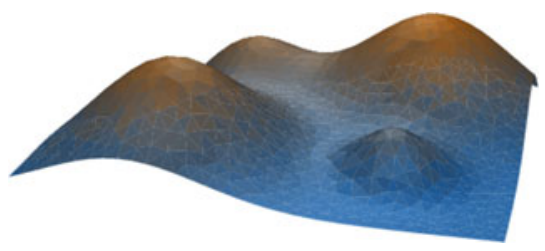

(a)

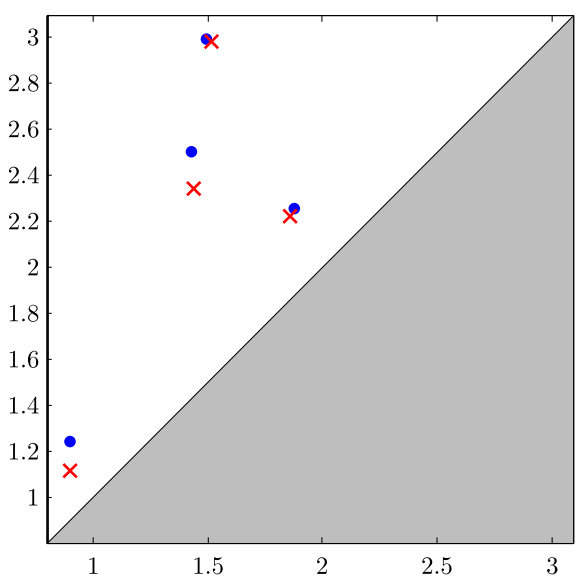

(c)

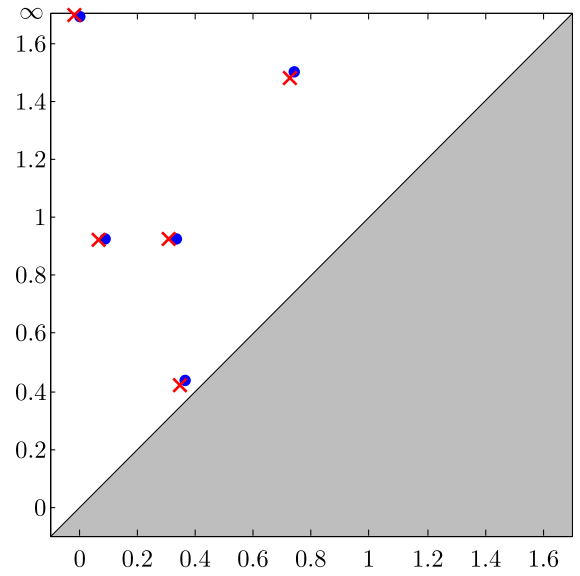

(b)

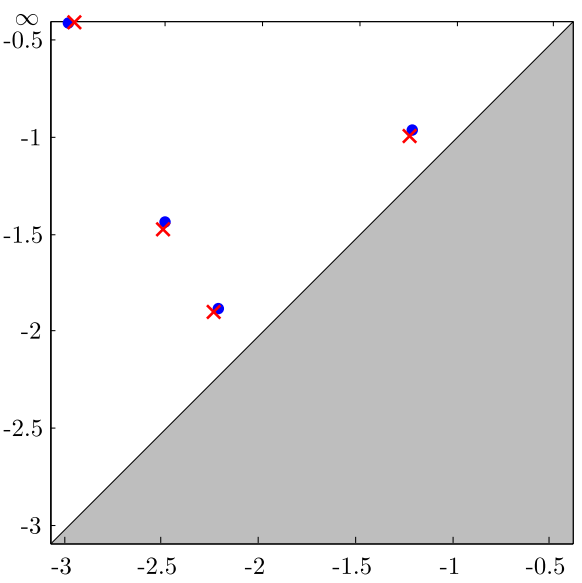

(d)

Fig. 2 (a) The 4 Gaussians dataset. The point cloud is a 0.05 -sample of the unit square. The zero-dimensional persistence diagram (b) and the one-dimensional persistence diagram (c). The (red) crosses mark the PD for the Rips parameter $\delta=0.052$, while blue points mark the PD of the input PL function. The opposite of the function has no one-dimensional feature, so we only show its zero-dimensional persistence diagram (d)

blue) and for the Rips complex with parameter $\delta=0.052$ (in red). Since we are on the plane, we compute the zero- and one-dimensional persistence diagrams, omitting the later when it is empty. Taking the PL functions as the "true" functions, we can see from the figures that the Rips diagrams do approximate the true diagrams.

In order to better quantify this proximity, we computed the bottleneck distances between the diagrams for the triangulations and various values of the Rips parameter $\delta$. Specifically we use $\delta=0.052,0.06,0.065$, and 0.07 . Since our ground-truth is a PL function, we can explicitly compute the Lipschitz constant $c$ from the triangulations. For the Gaussians, $c=4.625$, and for the crater, $c=7.34$. We show the results in Tables 2, 3, and 4. The first row shows the theoretical bound based on the com- 


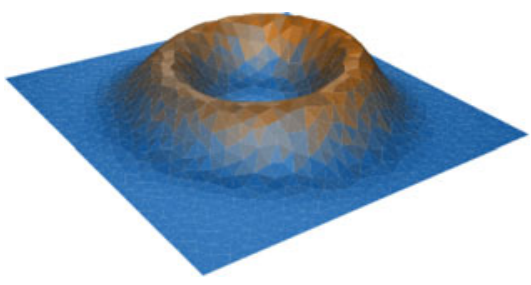

(a)

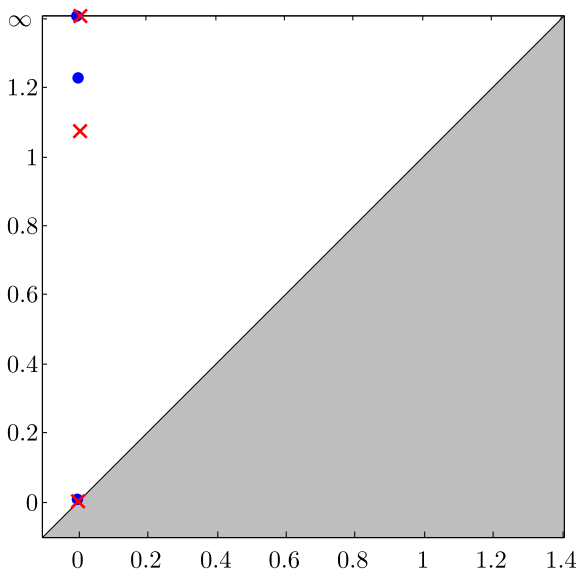

(b)

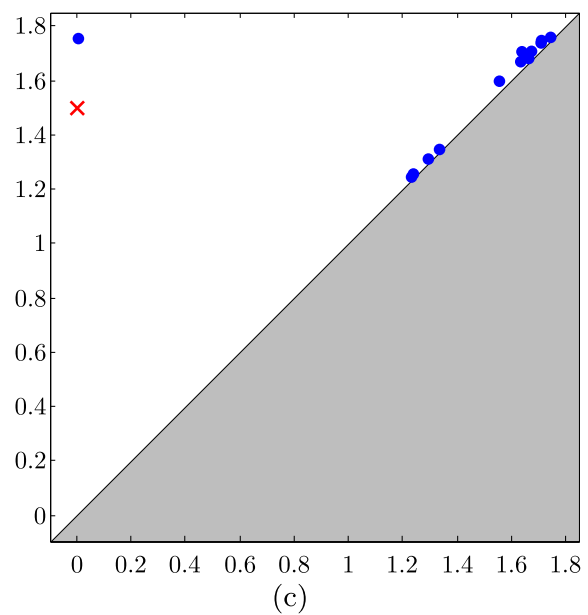

Fig. 3 (a) The crater-shaped PL function. The point cloud is a 0.05 -sample of the unit square. The zero-dimensional persistence diagram (b) and the one-dimensional persistence diagram (c). The (red) crosses mark the PD for the Rips parameter $\delta=0.052$, while blue points mark the PD of the input PL function

puted Lipschitz constants and the Rips parameter. For completeness, we show the distance matrix between all the diagrams. From the comparison of the Rips approximations (second row) it can be seen that the theoretical bound can be quite loose, but certainly holds. In general, the distance from the PL function increases with increasing Rips parameter. This is not required, however. For example, in the case of the negative of the Gaussians, the PL diagram is better approximated by $\delta=0.065$ than it is by $\delta=0.06$. For completeness, we show the distance matrix between all the diagrams. The distance between the Rips approximation also mostly increasing with the difference of the corresponding Rips parameters, which can be seen by the generally increasing values as we move away from the diagonal of the matrix. Note that although the proofs of Sect. 3 give a weaker interleaving between the various Rips 
Table 2 Bottleneck distance matrix and theoretical bound of the 4 Gaussians dataset for the input PL function and for various Rips parameters

\begin{tabular}{llllll}
\hline & PL & 0.052 & 0.06 & 0.065 & 0.07 \\
\hline Theoretical & 0 & 0.4808 & 0.5548 & 0.6011 & 0.6473 \\
PL & 0 & 0.1827 & 0.2262 & 0.2262 & 0.2910 \\
0.052 & 0.1827 & 0 & 0.0938 & 0.1047 & 0.1586 \\
0.06 & 0.2262 & 0.0938 & 0 & 0.0330 & 0.0648 \\
0.065 & 0.2262 & 0.1047 & 0.0330 & 0 & 0.0708 \\
0.07 & 0.2910 & 0.1586 & 0.0648 & 0.0708 & 0 \\
\hline
\end{tabular}

Table 3 Bottleneck distance matrix and theoretical bound of the 4 Gaussians dataset for the opposite of the input PL function and for various Rips parameters

\begin{tabular}{llllll}
\hline & PL & 0.052 & 0.06 & 0.065 & 0.07 \\
\hline Theoretical & 0 & 0.4808 & 0.5548 & 0.6011 & 0.6473 \\
PL & 0 & 0.0614 & 0.0781 & 0.0614 & 0.1571 \\
0.052 & 0.0614 & 0 & 0.0379 & 0.0333 & 0.0957 \\
0.06 & 0.0781 & 0.0379 & 0 & 0.0205 & 0.0790 \\
0.065 & 0.0614 & 0.0333 & 0.0205 & 0 & 0.0957 \\
0.07 & 0.1571 & 0.0957 & 0.0790 & 0.0957 & 0 \\
\hline
\end{tabular}

Table 4 Bottleneck distance matrix and theoretical bound of the crater-shaped dataset for the opposite of the input PL function and for various Rips parameters

\begin{tabular}{llllll}
\hline & PL & 0.052 & 0.06 & 0.065 & 0.07 \\
\hline Theoretical & 0 & 0.7639 & 0.8814 & 0.9549 & 1.0283 \\
PL & 0 & 0.2720 & 0.2865 & 0.2865 & 0.3279 \\
0.052 & 0.2720 & 0 & 0.1357 & 0.1357 & 0.1771 \\
0.06 & 0.2865 & 0.1357 & 0 & 0.0094 & 0.1359 \\
0.065 & 0.2865 & 0.1357 & 0.0094 & 0 & 0.1359 \\
0.07 & 0.3279 & 0.1771 & 0.1359 & 0.1359 & 0 \\
\hline
\end{tabular}

filtrations, experimentally, the bottleneck distance is dependent on the difference between the Rips parameters (in a specified range).

Note that the bottleneck distance is computed exactly by finding a minimumweight bipartite matching between the diagrams. Only a slight modification is needed to take into account the possible projections of the points onto the diagonal-see, e.g., [7] for details.

Clustering Clustering attempts to group points by assuming they are drawn from some unknown probability distribution. Our approach is inspired from mode-seeking 


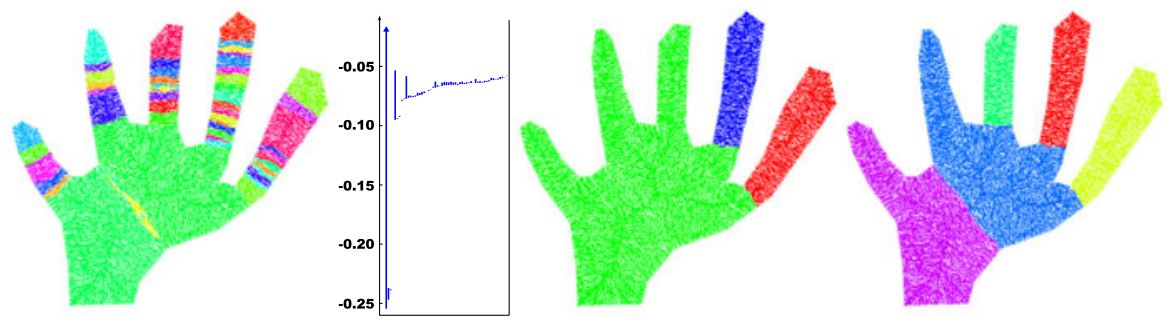

Fig. 4 Segmentation result on a sampled hand-shaped 2-D domain. The segmentation function is the Euclidean distance to the subset of the data points lying on the boundary of the domain. The barcode shows only three long intervals, corresponding to the palm of the hand and to the two rightmost fingers (center-right image), which have significant bottlenecks at their base. This suggests that the above function is not well suited for segmenting this type of shape. Indeed, when a finger (such as the index in our example) has no bottleneck, the exact distance to the boundary has no local maximum inside this finger, therefore no ascending region separates it from the rest of the hand. In practice, the inaccuracy of our gradient estimation creates artificial local maxima which, by chance, cover the fingers (left). However, our barcode reveals that their ascending regions are actually not persistent. The rightmost image shows the result obtained with a smaller persistence threshold $\tau$, which divides the palm of the hand before separating it from the index finger
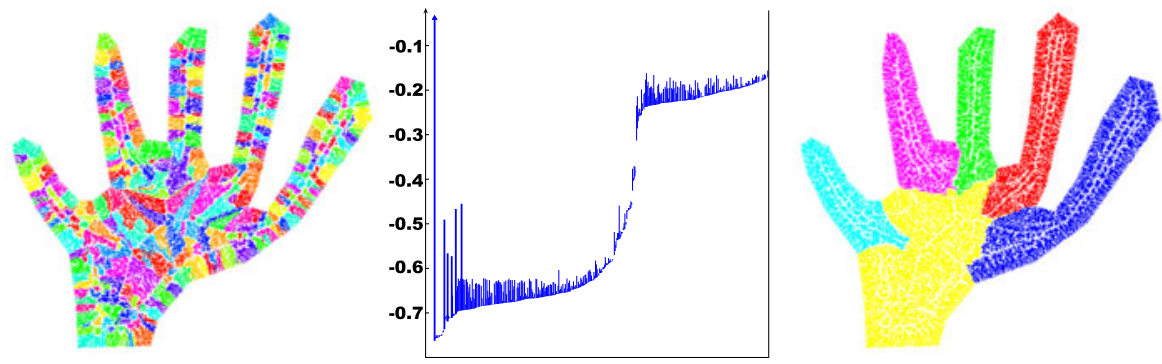

Fig. 5 Result obtained on the same data set as in Fig. 4, using the normalized diameter of the set of nearest boundary points as the segmentation function. The barcode shows six long intervals corresponding to the palm of the hand and to the five fingers. The results before and after merging nonpersistence clusters are shown respectively to the left and to the right of the barcode

techniques such as Mean-Shift [17]. Given an input point cloud $L$, we use a simple density estimator to approximate the local density at the points of $L$. As Fig. 8 shows, our estimator can be quite noisy. However, our emphasis is not on accurate density estimation, but rather on clustering with noisy density estimates. Our estimator is provided together with $L$ as input to the algorithm of Sect. 4.3, which clusters the points of $L$ according to the basins of attraction of the local maxima of the estimator in the Rips graph $G_{2 \delta}$ built over $L$. Due to the noisy nature of the estimator, we get a myriad of small clusters before the merging phase. The novelty of our approach is to provide visual feedback to the user in the form of an approximate persistence barcode of the estimator, from which the user can choose a relevant merging parameter $\tau$. For instance, the example of Fig. 8 is highly nonlinear and noisy, yet the barcode clearly shows two long intervals, suggesting that there are two main clusters. 

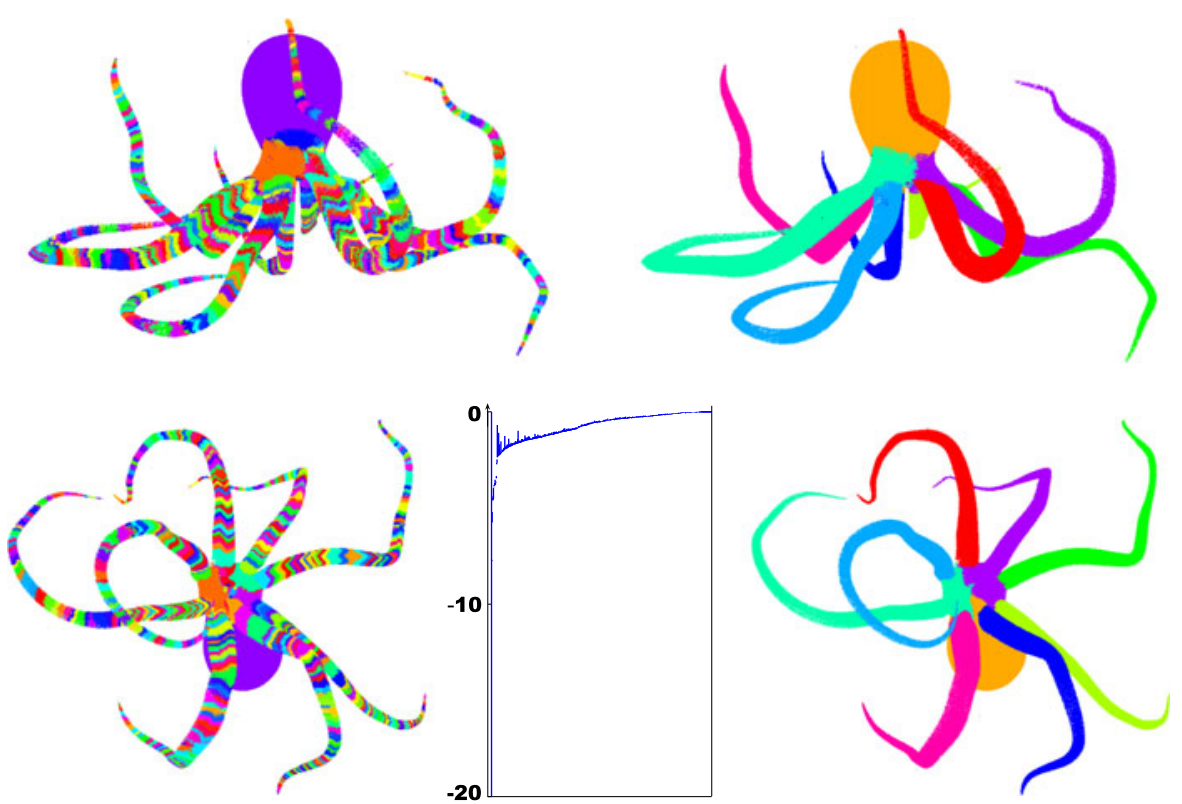

Fig. 6 Segmentation result obtained from a sampling of the interior of an octopus in 3-D. The segmentation function is the squared distance to the boundary of the body, whose barcode (center) somewhat emphasizes the bottlenecks at the base of the legs. With this segmentation function, there is a small range of values of the persistence threshold $\tau$ (easily computed from the barcode) that allow to recover the eight legs and the head. Pictures on the left show the result before merging clusters, while pictures on the right show the result after merging

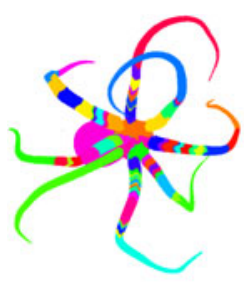

$\tau=0.1$

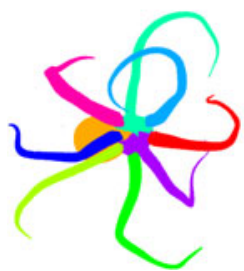

$\tau=0.5$

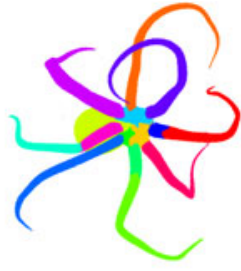

$\tau=0.2$

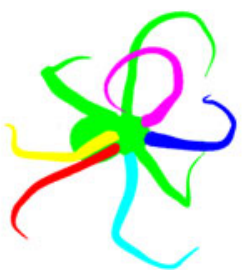

$\tau=0.6$

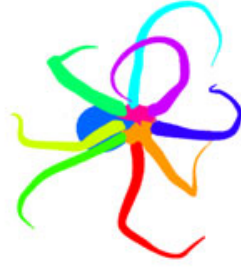

$\tau=0.3$

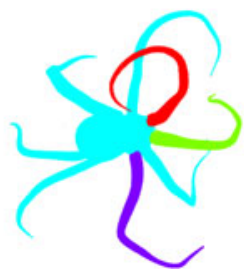

$\tau=0.7$

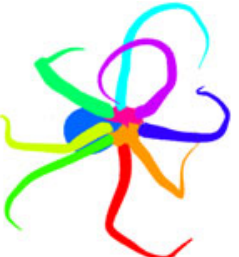

$\tau=0.4$

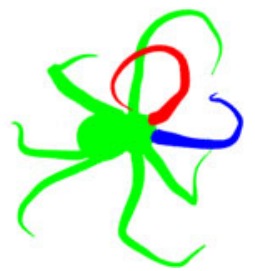

$\tau=0.8$

Fig. 7 Influence of the persistence threshold $\tau$ on the data set of Fig. 6 

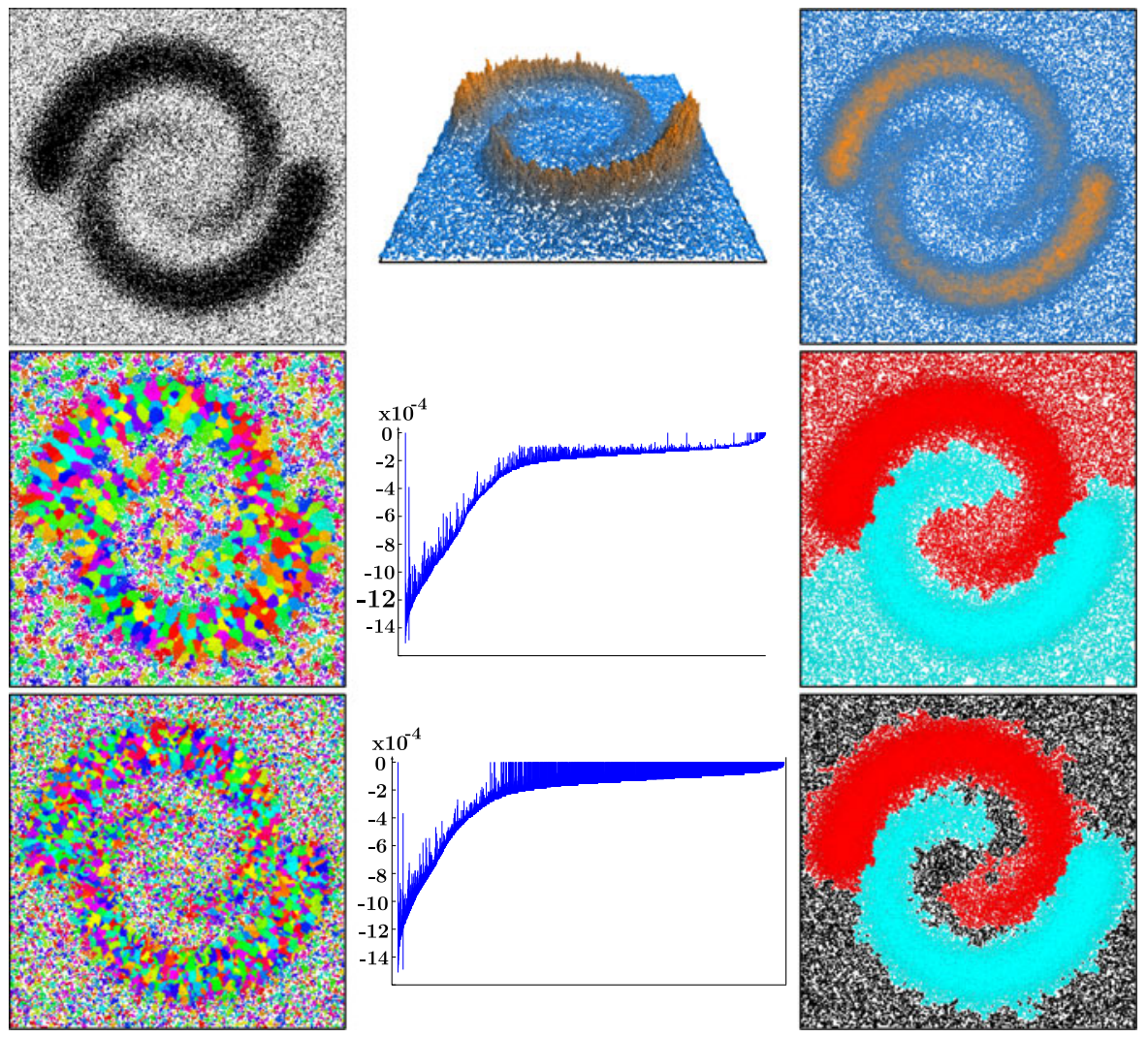

Fig. 8 A result in clustering. The top row shows the input provided to the algorithm of Sect. 4.3: the data points $(l e f t$ ), or rather their pairwise Euclidean distances, and the estimated density function $f$ (center and right). The 3-d view of $f$ illustrates how noisy this function can be in practice, thereby emphasizing the importance of our robustness results (Theorems 3 and 4). The two bottom rows show the results of the algorithm when applied to $-f$ to get the ascending regions of the maxima of $f$. Two different Rips parameters have been used, $\delta=15$ (center row) and $\delta=10$ (bottom row). Each row shows the result of the clustering before (left) and after (right) merging nonpersistent clusters. The persistence barcodes, shown in the center column, contain two prominent intervals (to the left) corresponding to the two main clusters. Since the estimated density is everywhere positive, the barcodes have been thresholded at 0 . Thus, intervals reaching 0 correspond to independent connected components in the Rips graph. The ones to the right of the barcodes are treated as noise and their corresponding clusters shown in black: this is because they appear lately, meaning that their corresponding peaks of $f$ are low

An important feature of our approach is to make a clear distinction between the merging criterion, governed by $\tau$ and based solely on persistence information, and the approximation accuracy of the basins of attraction of the maxima, governed by the Rips parameter $\delta$. In the example of Fig. 8, reducing $\delta$ while keeping $\tau$ fixed enabled us to separate the two spirals from the background while keeping them separate and integral.

Shape Segmentation The goal of shape segmentation is to partition a given shape into meaningful segments, such as fingers on a hand. This problem is ill-posed by na- 
ture, as meaningfulness is a subjective notion. Given a sampled shape $\mathbb{X}$, our approach is to apply the algorithm of Sect. 4.3 on some segmentation function $f: \mathbb{X} \rightarrow \mathbb{R}$ derived from the geometric features of $\mathbb{X}$. The output is a partition of the point cloud into clusters corresponding roughly to the basins of attraction of the significant peaks of $f$. Thus, we cast the segmentation problem into another problem, namely the one of finding a relevant segmentation function for a given class of data.

We investigated two functions in our experiments: distance to the set of samples lying on the boundary, as proposed in [18, 34], and diameter of the set of nearest samples on the boundary, normalized by the previous distance. We chose these two functions as a demonstration, but our method can be applied virtually with any segmentation function. The approximate barcodes computed by the algorithm provide information on the stability of the different segments. This information can be viewed as an indicator of the relevance of a given segmentation function on a particular class of data. In Figs. 4 and 5, the barcodes suggest that the second function is superior to the first one at separating the fingers from the palm of a hand. Yet, the second function turned out to be too noisy on the octopus data set of Figs. 6 and 7 .

\section{Final Remarks}

The potential of our approach stems from the observation that many problems can be reduced to the analysis of some scalar field defined over a given point cloud data. With the theoretical and algorithmic tools developed in this paper at hand, the users can cast these problems into the one of finding the scalar field that is most suitable for their particular purposes. Clustering is turned into a density estimation problem, and shape segmentation is turned into finding a relevant segmentation function for a given class of shapes. Many application-specific questions arise from this paradigm, which we do not pretend to solve in the paper. As mentioned in the introduction, we have begun working in this direction toward more complete solutions to specific applications. Particularly, we note that in [10], we address some of the specific issues involved in clustering. Notably, we extend the results to a probabilistic setting for when samples are drawn from a nonuniform distribution and begin to address the stability of the basins of attraction. We have also further explored the segmentation problem. In [30], we use the HKS function, introduced in [31], to compute mesh segmentations. The properties of the HKS combined with the persistence-based approach allow us to give stable segmentations that are invariant under isometric deformations. These two pieces of work have delved more deeply into specific applications introduced here, but there remain many interesting open questions.

Acknowledgements This work was carried out within the Associate Team TGDA: Topological and Geometric Data Analysis involving the Geometrica group at INRIA and the Geometric Computing group at Stanford University. It was further supported by the GIGA project ANR-09-BLAN-0331-01, DARPA grant HR0011-05-1-0007, and NSF grants ITR-0205671, FRG-0354543, and CCF-0634803.

\section{References}

1. http://www.cs.umd.edu/mount/ANN/ 
2. Attali, D., Glisse, M., Hornus, S., Lazarus, F., Morozov, D.: Persistence-sensitive simplification of functions on surfaces in linear time. In: Proc. TOPOINVIS (Topological Methods In Data Analysis and Visualization) (2009)

3. Bremer, P.-T., Edelsbrunner, H., Hamann, B., Pascucci, V.: Topological hierarchy for functions on triangulated surfaces. IEEE Trans. Vis. Comput. Graph. 10, 385-396 (2004)

4. Do Carmo, M.: Riemannian Geometry. Birkhäuser, Boston, Basel, Berlin (1992)

5. Cazals, F., Chazal, F., Lewiner, T.: Molecular shape analysis based upon the Morse-Smale complex and the Connolly function. In: Proc. 19th Annu. ACM Sympos. Comput. Geom, pp. 237-246 (2003)

6. Chazal, F., Cohen-Steiner, D., Guibas, L.J., Glisse, M., Oudot, S.Y.: Proximity of persistence modules and their diagrams. In: Proc. 25th ACM Sympos. Comput. Geom. (2009)

7. Chazal, F., Cohen-Steiner, D., Guibas, L.J., Mémoli, F., Oudot, S.Y.: Gromov-Hausdorff stable signatures for shapes using persistence. In: Computer Graphics Forum (Proc. SGP 2009), pp. 1393-1403 (2009)

8. Chazal, F., Cohen-Steiner, D., Lieutier, A.: A sampling theory for compact sets in Euclidean space. In: Proc. 22nd Annual Sympos. Comput. Geom, pp. 319-326 (2006)

9. Chazal, F., Guibas, L.J., Oudot, S.Y., Skraba, P.: Analysis of scalar fields over point cloud data. In: Proc. 20th ACM-SIAM Sympos. Discrete Algorithms, pp. 1021-1030 (2009)

10. Chazal, F., Guibas, L.J., Oudot, S.Y., Skraba, P.: Persistence-based clustering in Riemannian manifolds. Research Report 6968, INRIA, June 2009

11. Chazal, F., Lieutier, A.: Stability and computation of topological invariants of solids in $\mathbb{R}^{n}$. Discrete Comput. Geom. 37(4), 601-617 (2007)

12. Chazal, F., Oudot, S.Y.: Towards persistence-based reconstruction in Euclidean spaces. In: Proc. 24th ACM Sympos. Comput. Geom, pp. 232-241 (2008)

13. Cohen-Steiner, D., Edelsbrunner, H., Harer, J.: Stability of persistence diagrams. In: Proc. 21st ACM Sympos. Comput. Geom, pp. 263-271 (2005)

14. Cohen-Steiner, D., Edelsbrunner, H., Harer, J., Morozov, D.: Persistent homology for kernels and images. Preprint (2008)

15. Cohen-Steiner, D., Edelsbrunner, H., Morozov, D.: Vines and vineyards by updating persistence in linear time. In: Proc. 22nd Sympos. on Comput. Geom., pp. 119-126 (2006)

16. Collins, A., Zomorodian, A., Carlsson, G., Guibas, L.J.: A barcode shape descriptor for curve point cloud data. Comput. Graph. 28(6), 881-894 (2004)

17. Comaniciu, D., Meer, P.: Mean shift: a robust approach toward feature space analysis. IEEE Trans. Pattern Anal. Mach. Intell. 24(5), 603-619 (2002)

18. Dey, T.K., Wenger, R.: Stability of critical points with interval persistence. Discrete Comput. Geom. 38, 479-512 (2007)

19. Edelsbrunner, H., Harer, J., Zomorodian, A.: Hierarchical Morse complexes for piecewise linear 2manifolds. In: Proc. 17th Annual Sympos. Comput. Geom., pp. 70-79 (2001)

20. Edelsbrunner, H., Letscher, D., Zomorodian, A.: Topological persistence and simplification. Discrete Comput. Geom. 28, 511-533 (2002)

21. Edelsbrunner, H., Morozov, D., Pascucci, V.: Persistence-sensitive simplification of functions on 2manifolds. In: Proc. 22nd Sympos. on Comput. Geom., pp. 127-134 (2006)

22. Gao, J., Guibas, L., Oudot, S., Wang, Y.: Geodesic Delaunay triangulation and witness complex in the plane. Full version, partially published in Proc. 18th ACM-SIAM Sympos. on Discrete Algorithms, pp. 571-580 (2008). Full draft available at: http://graphics.stanford.edu/projects/lgl/papers/ ggow-gtwcp-08/ggow-gdtwcp-08-full.pdf

23. Guibas, L.G., Oudot, S.Y.: Reconstruction using witness complexes. In: Proc. 18th Sympos. on Discrete Algorithms, pp. 1076-1085 (2007)

24. Guibas, L.J.: Kinetic data structures - a state of the art report. In: Agarwal, P.K., Kavraki, L.E., Mason, M. (eds.) Proc. Workshop Algorithmic Found. Robot, pp. 191-209. A.K. Peters, Wellesley (1998)

25. Gyulassy, A., Natarajan, V., Pascucci, V., Bremer, P.-T., Hamann, B.: Topology-based simplification for feature extraction from 3d scalar fields. In: Proc. IEEE Conf. Visualization, pp. 275-280 (2005)

26. Gyulassy, A., Natarajan, V., Pascucci, V., Bremer, P.-T., Hamann, B.: A topological approach to simplification of three-dimensional scalar fields. IEEE Trans. Vis. Comput. Graph. 12(4), 474-484 (2006)

27. Hatcher, A.: Algebraic Topology. Cambridge University Press, Cambridge (2001)

28. Milnor, J.W.: Morse Theory. Princeton University Press, Princeton (1963)

29. Niyogi, P., Smale, S., Weinberger, S.: Finding the homology of submanifolds with high confidence from random samples. Discrete Comput. Geom. 39(1), 419-441 (2008) 
30. Skraba, P., Ovsjanikov, M., Chazal, F., Guibas, L.J.: Persistence-based segmentation of deformable shapes. In: Third Workshop on Non-Rigid Shape Analysis and Deformable Image Alignment (2010)

31. Sun, J., Ovsjanikov, M., Guibas, L.: A concise and provably informative multi-scale signature based on heat diffusion. In: Eurographics Symposium on Geometry Processing (SGP) (2009)

32. Tenenbaum, J.B., de Silva, V., Langford, J.C.: A global geometric framework for nonlinear dimensionality reduction. Science 290(5500), 2319-2323 (2000)

33. Zhao, F., Guibas, L.J.: Wireless Sensor Networks. Morgan Kaufmann, San Mateo (2004)

34. Zhu, X., Sarkar, R., Gao, J.: Shape segmentation and applications in sensor networks. In: Proc. INFOCOM, pp. 1838-1846 (2007)

35. Zomorodian, A., Carlsson, G.: Computing persistent homology. Discrete Comput. Geom. 33(2), 249274 (2005) 